\title{
Water Adlayers on Noble Metal Surfaces: Insights from Energy Decomposition Analysis
}

\author{
Paul Clabaut, Ruben Staub, Joachim Galiana, Elise Antonetti, Stephan Steinmann
}

Submitted date: 07/05/2020 - Posted date: 08/05/2020

Licence: CC BY-NC-ND 4.0

Citation information: Clabaut, Paul; Staub, Ruben; Galiana, Joachim; Antonetti, Elise; Steinmann, Stephan (2020): Water Adlayers on Noble Metal Surfaces: Insights from Energy Decomposition Analysis. ChemRxiv. Preprint. https://doi.org/10.26434/chemrxiv.12263117.v1

Water molecules adsorbed on noble metal surfaces are of fundamental interest in surface science, heterogeneous catalysis and as a model for the metal/water interface. Herein, we analyse 27 water structures adsorbed on five noble metal surfaces $(\mathrm{Cu}, \mathrm{Ag}, \mathrm{Au}, \mathrm{Pd}, \mathrm{Pt})$ via density functional theory and energy decomposition analysis based on the block localized wave function technique. The structures, ranging from the monomers to ice adlayers, reveal that the charge-transfer from water to the surface is nearly independent from the charge-transfer between the water molecules, while the polarization energies are cooperative. Dense water-water networks with small surface dipoles, such as the sqrt(39) x sqrt(39) unit cell (experimentally observed on $\mathrm{Pt}(111)$ ) are favored compared to the highly ordered and popular $\mathrm{H}^{\text {up }}$ and $\mathrm{H}^{\text {down }}$ phases. The second main result of our study is that the many-body interactions, which stabilize the water assemblies on the metal surfaces, are dominated by the polarization energies, with the charge-transfer scaling with the polarization energies. Hence, if an empirical model could be found that reproduces the polarization energies, the charge-transfer could be predicted as well, opening exciting perspectives for force field development.

File list (3)

WaterLayers_Main.pdf (749.93 KiB)

view on ChemRxiv • download file

WaterLayers_SI.pdf (282.38 KiB) view on ChemRxiv - download file imecs.tar.gz (14.15 KiB) view on ChemRxiv - download file 


\title{
Water Adlayers on Noble Metal Surfaces: Insights from Energy Decomposition Analysis
}

\author{
Paul Clabaut, ${ }^{1}$ Ruben Staub, ${ }^{1}$ Joachim Galiana, ${ }^{1}$ Elise Antonetti, ${ }^{1}$ and Stephan N. Steinmann ${ }^{1}$ \\ Univ Lyon, Ecole Normale Supérieure de Lyon, CNRS Université Lyon 1, Laboratoire de Chimie UMR 5182, 46 allée d'Italie, \\ F-69364, LYON, France
}

(Dated: 7 May 2020)

\begin{abstract}
Water molecules adsorbed on noble metal surfaces are of fundamental interest in surface science, heterogeneous catalysis and as a model for the metal/water interface. Herein, we analyse 27 water structures adsorbed on five noble metal surfaces $(\mathrm{Cu}, \mathrm{Ag}, \mathrm{Au}, \mathrm{Pd}, \mathrm{Pt})$ via density functional theory and energy decomposition analysis based on the block localized wave function technique. The structures, ranging from the monomers to ice adlayers, reveal that the charge-transfer from water to the surface is nearly independent from the charge-transfer between the water molecules, while the polarization energies are cooperative. Dense water-water networks with small surface dipoles, such as the $\sqrt{39} \times \sqrt{39}$ unit cell (experimentally observed on $\mathrm{Pt}(111)$ ) are favored compared to the highly ordered and popular $\mathrm{H}^{\mathrm{up}}$ and $\mathrm{H}^{\text {down }}$ phases. The second main result of our study is that the many-body interactions, which stabilize the water assemblies on the metal surfaces, are dominated by the polarization energies, with the charge-transfer scaling with the polarization energies. Hence, if an empirical model could be found that reproduces the polarization energies, the charge-transfer could be predicted as well, opening exciting perspectives for force field development.
\end{abstract}

\section{INTRODUCTION}

Ice-like water layers over noble metal surfaces are widely studied, both experimentally and theoretically. ${ }^{1-4}$ Due to the sparsity of the characterization of the metal/water interface, they are sometimes considered model systems for the solid/liquid interface, ${ }^{5-9}$ even though the validity of this extrapolation is far from obvious. ${ }^{10,11}$ Furthermore, the ice adlayers are regularly used to model the metal/liquid interface in (electro-)catalysis. ${ }^{12-16}$ The alternatives for approximate treatements of the solvent are implicit solvents, ${ }^{17}$ which do not compete with adsorbates for surface sites, ${ }^{18}$ microsolvation ${ }^{19,20}$ which solvates adsorbates only locally, and ab initio molecular dynamics, which is computationally very expensive ${ }^{21}$.

The most commonly reported and applied ice adlayers over closed-packed noble-metal surfaces are the $\sqrt{3} \times \sqrt{3} \mathrm{H}^{\text {up }}$ and $\mathrm{H}^{\text {down }}$ models, going back to the seminal STM work of Doering on $\mathrm{Ru} .{ }^{1}$ However, larger unit cells have been observed for $\operatorname{Pt}(111)^{2}$ and explained in terms of more disordered ice-like layers featuring ring-structures of various sizes. ${ }^{3}$

Previous theoretical studies have focused on the bonding mechanism of individual monomers on metal surfaces ${ }^{22,23}$ or on the possibility of water dissociation. ${ }^{24}$ Herein, we focus on non-dissociated water layers, fully covering the noble metal surfaces. The purpose of this study is, on the one hand, to elucidate the relative stability of these ice-like structures on five noble metal surfaces $(\mathrm{Cu}, \mathrm{Pd}, \mathrm{Ag}, \mathrm{Pt}, \mathrm{Au})$ and, on the other hand, to identify the driving force of their formation via energy decomposition analysis. We rely on dispersion corrected DFT to achieve a balanced description between water-water and water-metal interactions. ${ }^{25,26}$

Energy decomposition analysis (EDA) is a powerful tool which is mostly applied in molecular chemistry, ${ }^{27-29}$ but also increasingly in condensed phase ${ }^{30}$ and at surfaces. ${ }^{31-33}$ In particular, we have recently extended the block localized wave function (BLW) technique ${ }^{34-36}$ to metallic surfaces. ${ }^{33}$ The BLW based EDA now allows to decompose the adsorption energy into four terms: deformation, frozen, polarization and charge-transfer, which encompasses electron sharing. This energy decomposition not only provides deep insight into the bonding, but also allows to gain information for force field development: ${ }^{37}$ The charge-transfer (chemisorption) is the term that is the most difficult to reproduce, as it is intrinsically a many-body term with no generally applicable analytical expression known for it. The polarization is, on the other hand, a better understood many-body term, which can be modelled via induced dipoles, themselves modelled according to different techniques. BLW, which includes polarization at the DFT level, also defines the limit of which precision can be expected from a polarizable force field in the absence of error cancellations between different interaction energy components.

To achieve this detailed insight, the remaining of the work is structured as follows: After describing the computational details, we analyse the stability of the various ice-layers on the five investigated metal surfaces. Next, we perform an EDA for the ice-layers, but also for 23 smaller (monomer to heptamer) clusters. According to these computations, the polarization interaction is strongly correlated to the charge-transfer energy, so that the total interaction can be estimated based on the (linear-scaling ${ }^{38}$ ) BLW energy. Furthermore, we quantify the cooperativity between water-water and water-metal polarization interactions and evidence a competition between the water-water and water-metal charge-transfer interactions.

\section{METHODS}

We start by defining the total adsorption energy of a given system:

$$
\Delta E_{a d s}=E_{S C F}-E_{\text {surf }}^{o p t}-n \cdot E_{W^{o p t}}+\Delta E_{B S S E}
$$

where $E_{S C F}$ is the standard KS-SCF energy of the full system, $E_{\text {surf }}{ }^{\text {opt }}$ and $E_{W^{\text {opt }}}$ are the corresponding energies of the freely optimized surface and water molecule, respectively. $n$ is the number of water molecules in a given system. Since the BLW is only defined in a localized basis set, we have to correct for the 
basis set superposition error (BSSE), which we do according to the counterpoise procedure of Boys and Bernardi ${ }^{39}$, giving rise to the (by definition positive) energy correction $\triangle E_{B S S E}$.

As common in BLW-EDA, ${ }^{27,40,41}$ we decompose the total adsorption energy $\Delta E_{a d s}$ into:

$$
\Delta E_{a d s}=\Delta E_{\text {deform }}+\Delta E_{\text {frozen }}+\Delta E_{\text {pol }}+\Delta E_{C T}
$$

where $\Delta E_{\text {deform }}$ is the preparation or deformation energy, $\Delta E_{\text {frozen }}$ is the frozen energy term that describes the interaction of the isolated fragment densities brought together and covers electrostatic interaction and Pauli repulsion ${ }^{27}$ as well as dispersion interactions ${ }^{41} . \Delta E_{p o l}$ is the polarization energy which is obtained by variationally optimizing the BLW. $\Delta E_{C T}$ is, finally, the charge transfer interaction that includes the covalent bond formation. Note, that the BSSE only affects the charge-transfer term, as all other terms are evaluated using the same fragment-decomposed basis set.

The following equation summarizes the scheme and different terms. Further details on the computation of these terms are given in the corresponding equations as indicated:

$$
E_{\text {isolated }} \underbrace{\frac{\left.\Delta E_{\text {for }}\right)}{\longrightarrow} E_{\text {fragments }}}_{\Delta E_{\text {deform }}(E q .2)} \underbrace{\overbrace{\frac{\Delta E_{\text {frozen }}}{E q .4 b} E_{S F D} \frac{\Delta E_{\text {int }}^{B L W}}{E q .4 c}(E q .6)} E_{B L W} \frac{\Delta E_{C T}}{E q .4 d}}_{\Delta E_{\text {int }}(E q .5)} E_{c o r} \stackrel{-\Delta E_{B S S E}}{\longrightarrow} E_{S C F}
$$

where $E_{\text {isolated }}=E_{\text {surfopt }}+n \cdot E_{W_{i}^{\text {opt }}}$ is the sum of the electronic energy of each fragment optimized separately, $E_{\text {fragments }}=$ $E_{\text {surf }}$ sys $+\sum_{i}^{n} E_{W_{i}^{s y s}}$ is the sum of the energy of each fragment evaluated in its final geometry. The superscript "sys" corresponds to the energy of a fragment in the geometry adopted in the presence of the other fragment. $E_{S F D}$ is the total energy after Superposition of the Fragment Densities, $E_{B L W}$ is the total energy obtained by the Block Localized Wavefunction ${ }^{33}$ and $E_{c o r}$ corresponds to the final energy of the complete system, corrected for the BSSE, while $E_{S C F}$ is the energy obtained by a standard SCF computation.

This leads to the following definitions for the four terms of the adsorption energy as decomposed in Eq. 2:

$$
\begin{aligned}
\Delta E_{\text {deform }} & =E_{\text {surf } f^{s y s}}-E_{\text {surfopt }}+\sum_{i}^{n} E_{W_{i}^{\text {sys }}}-n \cdot E_{W^{o p t}} \\
\Delta E_{\text {frozen }} & =E_{S F D}-E_{\text {surf,sys }}-\sum_{i}^{n} E_{W_{i}^{\text {sys }}} \\
\Delta E_{\text {pol }} & =E_{B L W}-E_{S F D} \\
\Delta E_{C T} & =E_{S C F}-E_{B L W}+\Delta E_{B S S E}
\end{aligned}
$$

Furthermore, we define the interaction energy, $\Delta E_{\text {int }}$ as the adsorption energy excluding the deformation energy, i.e.,

$$
\Delta E_{\text {int }}=\Delta E_{\text {frozen }}+\Delta E_{\text {pol }}+\Delta E_{C T}
$$

Similarly, we define the BLW interaction energy as the interaction energy that excludes the charge-transfer interaction:

$$
\Delta E_{B L W}=\Delta E_{\text {frozen }}+\Delta E_{p o l}
$$

Since $\Delta E_{B L W}$ include all polarization contributions at the DFT level but excludes any charge-transfer, it can be understood as the interaction energy of an "ideal" polarizable force field. Furthermore, computationally its evaluation can be performed with near linear scaling with respect to the number of fragments, ${ }^{38}$ which contrasts with the cubic scaling for the computations including the charge-transfer interactions.

For the energy decomposition analysis and its interpretation, each system is either divided into two blocks (one for the metallic surface, one for all the $n$ water molecules together) or into $n+1$ blocks.

Taking the frozen interaction as an example, we denote the standard decomposition:

$$
\Delta E_{\text {frozen }}=\Delta E_{\text {frozen }}\left(W_{1}, \ldots, W_{n}, \text { surf }\right)
$$

as the situation where every water molecule $W_{i}$ is treated as a separate subsystem. This contrasts with decomposition into two blocks, the surface and the adlayer:

$$
\Delta E_{\text {frozen }}^{\text {surf-layer }}=\Delta E_{\text {frozen }}\left(\bigcup_{i} W_{i}, \text { surf }\right)
$$

where all the water molecules are treated together as a single block and the surface is a second block. Finally, in order to assess many-body effects, we also determine the "additive" frozen interaction:

$$
\Delta E_{\text {frozen }}^{a d d}=\sum_{i}^{n} \Delta E_{\text {frozen }}\left(W_{i}, \text { surf }\right)
$$

where we perform $n$ separate computations, one for each water molecule, and then sum the corresponding contributions.

The standard decomposition leads to the most complete interaction while Eq. 8 excludes the water-water interaction components and Eq. 9 is free of any many-body interactions. It is, therefore, possible to define the missing part of the interaction component

$$
\Delta E_{\text {frozen }}^{\text {NonAdd }}=\Delta E_{\text {frozen }}-\Delta E_{\text {frozen }}^{a d d}
$$

which represent the non-additive part of the interaction.

Analogous equations to Eq. 7-10 can be written for the polarization and charge-transfer energy. 


\section{COMPUTATIONAL DETAILS}

The adsorbed structures were optimized with VASP 5.4.1 $1^{42,43}$ using periodic boundary conditions applying the re-optimized Perdew, Burke and Ernzerhofer (PBE) functional to make it compatible with the non-local van der Waals (vdW) functional, in short optPBE-vdW ${ }^{44}$ functional. An energy cutoff of $400 \mathrm{eV}$ is chosen for the expansion of the plane-wave basis set. The electron-ion interactions are described by the PAW formalism. ${ }^{45,46}$ The unit cells are built from bulk platinum (2.821 $\AA$ nearest neighbor distance) with four metallic layers. The out-of-plane vector of the unit cell was chosen to be $\sim 20 \AA$ to achieve a negligible interaction between periodic images. Coordinate files for all discussed systems are available in the supporting information.

In $\mathrm{CP} 2 \mathrm{~K},{ }^{47,48}$ the molecular orbitals were represented by a double- $\zeta$ Gaussian basis set with one set of polarization functions, called DZVP-MOLOPT-SR-GTH for both BLWEDA and BSSE corrected SCF DFT simulations. ${ }^{49}$ A cutoff of $400 \mathrm{Ry}$ was used to describe the electron density. The exchange-correlation (XC) energy was approximated with the optPBE-vdW ${ }^{44}$ functional. Like in VASP, the Brillouin zone was described at the $\Gamma$-point. Goedecker, Teter and Hutter (GTH) pseudo-potentials ${ }^{50}$ based on the PBE functional were used to describe the interactions between the valence electrons and the ionic cores, and the electronic smearing was approximated by a Fermi-Dirac distribution at $300 \mathrm{~K}$. As discussed in our previous publication ${ }^{33}$ the 18 valence electron potential is necessary for Pt to obtain similar results between $\mathrm{CP} 2 \mathrm{~K}$ and VASP. For $\mathrm{Cu}$ and $\mathrm{Au}$ the 11 valence electron potential is applied. For the adopted choice, Fig. S1 provides the comparison between $\mathrm{CP} 2 \mathrm{~K}$ and VASP, showing an excellent agreement.

In order to identify the water molecules in the ice-layers, where the atoms are ordered by elements rather than molecule, we have used our in-house code imecs, which is provided in the supplementary information.

\section{RESULTS AND DISCUSSION}

\section{A. Relative Stability of Ice-like Layers}

We are comparing five previously reported ice-like layers (depicted in Fig. 1) on five metals, which we will denote $\mathrm{H}^{\text {up }}$, $\mathrm{H}^{\text {down }}$, chain-H $\mathrm{H}^{\text {down }}, \sqrt{37}$ and $\sqrt{39}$. The nominal coverage of these structures is $0.67 \mathrm{ML}$ for the first three, 0.70 and 0.72 ML for the last two. Figure 2 shows that the $\sqrt{39}$ structure leads to the lowest surface energy $\Gamma$ for almost all metals, closely followed by $\sqrt{37} \cdot \mathrm{Cu}(111)$ is the exception in the sense that it is the only metal investigated herein for which $\Gamma(\sqrt{37})<\Gamma(\sqrt{39})$. Concomitantly, $\mathrm{Cu}(111)$ has the lowest interatomic distance of $2.58 \AA$ which compares to $2.80 \AA$ for $\mathrm{Pd}$, which is the second smallest metal investigated here.

In terms of absolute values, the surface energy of $\operatorname{Pt}(111)$ for the $\sqrt{37}$ structure is $1.73 \mathrm{kcal} /\left(\mathrm{mol} \cdot \AA^{2}\right)$, which compares to $0.46 \mathrm{kcal} /\left(\mathrm{mol} \cdot \AA^{2}\right)$ for the adhesion of solid water at $\sim 100$ $\mathrm{K}^{51}$ and $3.45 \mathrm{kcal} /\left(\mathrm{mol} \cdot \AA^{2}\right)$ for the Pt/liquid water tension. ${ }^{52}$ For a broader comparison to experiment, Fig. S2 shows the cor- relation between the lowest surface energy of each metal and the reported experimental value. Except for $\mathrm{Cu}$, the trends are nicely reproduced. Since, furthermore, the interatomic distance of $\mathrm{Cu}$ is significantly smaller compared to the other metals and thus less compatible with the ideal H-bond length of $\sim 2.8$ $\AA$, this might indicate that liquid water behaves differently on $\mathrm{Cu}(111)$ compared to the other noble metal surface.

\section{B. Electronic Analysis}

Before moving to the energy decomposition analysis, we here investigate the electronic nature of the various interfaces by computing the surface dipole moment and the workfunction $\Phi$. The workfunction is intimately connected to the electrochemical potential and it has been argued that the $\mathrm{H}^{\mathrm{up}}$ and $\mathrm{H}^{\text {down }}$ phases should co-exist over large potential ranges. ${ }^{9,10}$ However at that time the three other surfaces investigated here have not been assessed.

All ice adlayers taken alone, except $\mathrm{H}^{\text {up }}$ for all metals and chain- $\mathrm{H}^{\text {down }}$ on top of $\mathrm{Cu}(111)$, feature a positive dipole moment, meaning that there is a positive charge accumulation on the "bottom" and a negative one on the "top" (see SI). The maximum (1.4 e $\AA$ ) is obtained for $\sqrt{39}$ over $\operatorname{Pd}(111)$, while the minimum is found for $\mathrm{H}^{\text {up }}$ over $\mathrm{Cu}(111)(-2.7 \mathrm{e} \AA)$. The water layers, when optimized on different metallic surfaces (and hence, on different lattice size), undergo noticeable geometry distortions. A specific layer, evaluated without metallic slab but in the perturbed geometry corresponding to different metals, can exhibit a range of dipole moment up to $1.3 \mathrm{e} \AA$. This maximum is obtained for the $\mathrm{H}^{\mathrm{up}}$ layer optimized on $\mathrm{Au}(111)$ $(-1.4 \mathrm{e} \AA)$ compared to the one from $\mathrm{Cu}(111)(-2.7 \mathrm{e} \AA)$. Since water is adsorbed only slightly stronger on $\mathrm{Cu}$ than on $\mathrm{Au}(111)$, this shows that it is mostly the lattice missmatch, and not so much the interaction strength with the metal, that affects the electronic structures via geometrical constraints significantly.

Similarly, the change in workfunction upon adsorption of an ice adlayer depends significantly on the metal (larger changes in absolute values for $\mathrm{Pt}, \mathrm{Pd}$ and $\mathrm{Cu}$ than for $\mathrm{Ag}$ and $\mathrm{Au}$ ) and on the ice layer (see Fig. 3). In particular, the workfunction is lowered by almost $3 \mathrm{eV}$ when adsorbing the $\mathrm{H}^{\text {up }}$ layer on $\mathrm{Pt}, \mathrm{Pd}$ and $\mathrm{Cu}$, but "only" $1.6 \mathrm{~V}$ on $\mathrm{Au}$ and $\mathrm{Ag}$. Given the very reductive nature of the $\mathrm{H}^{\text {up }}$ structure, ${ }^{9}$ its stability is doubtful in itself. Even though one could have expected that the $\mathrm{H}^{\text {down }}$ layer has the opposite effect, this is not the case and the workfunction still drops for $\mathrm{Pt}$ and $\mathrm{Pd}(-0.8 \mathrm{eV})$, but remains unchanged for $\mathrm{Cu}$ and increases slightly $(0.2 \mathrm{eV})$ for $\mathrm{Ag}$ and $\mathrm{Au}$. This not only shows that a purely geometric analysis of the structure is not enough to retrieve the trends on the electronic structure, but also that the metal-dependant interaction plays a major role.

As expected based on basic physical principles, ${ }^{53}$ the surface dipole moments of the hydrated metal surfaces are correlated with the change in workfunction $(\Delta \Phi)$, with an intercept of zero (see Fig. S3). In other words, a positive surface dipole moment is associated with a positive change in workfunction and vice versa. Given the generally positive dipole moments for the isolated layers as discussed above, the dominance of 


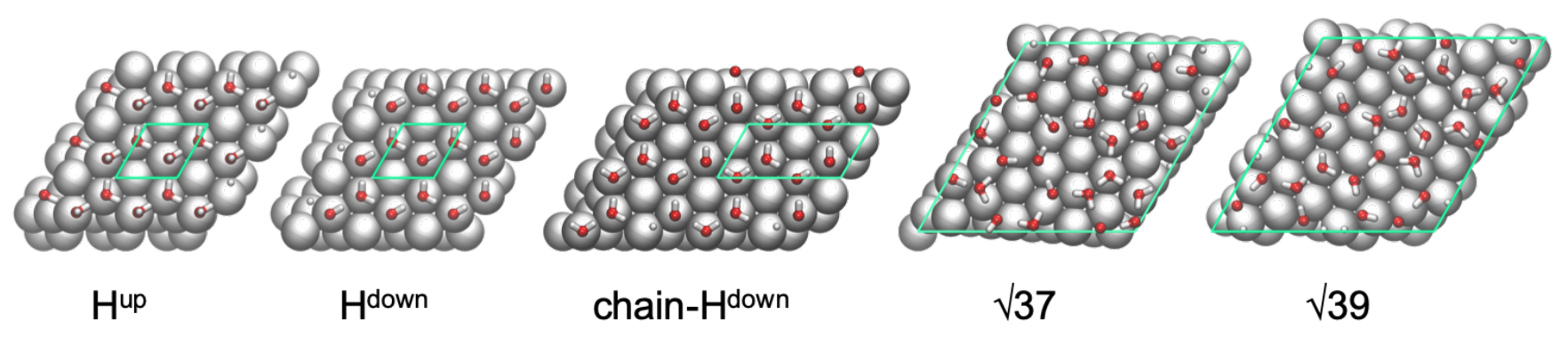

FIG. 1: Structures of the ice adlayers on $\operatorname{Pt}(111)$ together with their short-hand notation as used herein. The unit cell is indicated in green. For the small unit cells, a supercell is chosen to have comparable sizes for all systems and allow the use of the $\Gamma$ point only in the DFT computations.

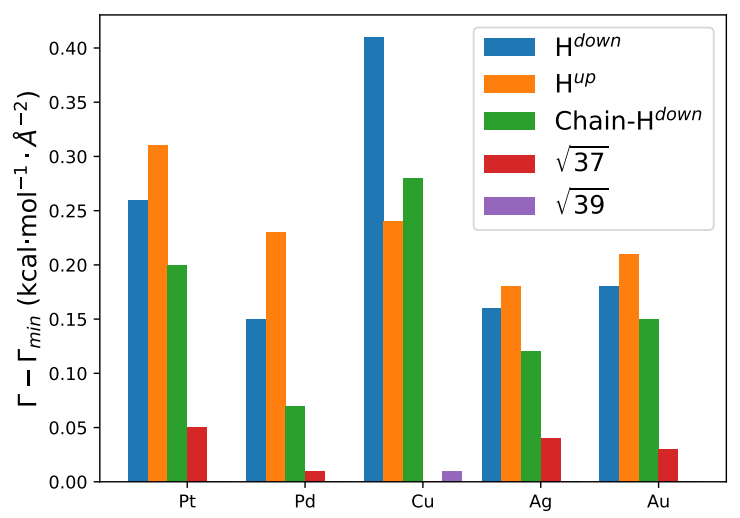

FIG. 2: Surface energies $\Gamma$ in $\mathrm{kcal} / \mathrm{mol} / \AA^{2}$ relative to the most stable ice adlayer, i.e., $\sqrt{39}$ for all metals except for $\mathrm{Cu}$, where $\sqrt{37}$ is slightly more stable. The higher the bar, the less stable is the corresponding structure.

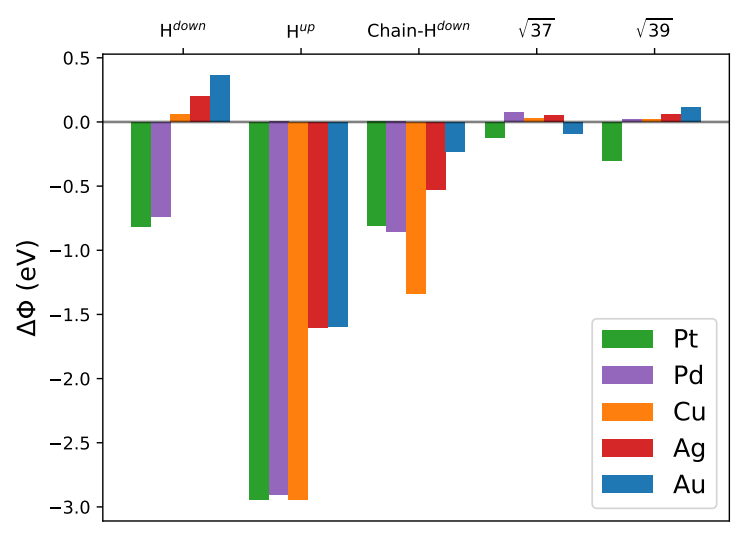

FIG. 3: The change of workfunction $(\Delta \Phi)$ when an ice adlayer is adsorbed on a (111) noble metal surface. negative changes in $\Phi$, and, thus, the negative dipole moments for hydrated surfaces, require additional explanations. Indeed, the change in dipole moment upon adsorption is generally negative (the one exception being $\mathrm{H}^{\text {up }}$ on $\mathrm{Cu}(111)$ ), with an average of $-0.8 \mathrm{e} \AA$ and a minimum of $-1.6 \mathrm{e} \AA$ (chain- $\mathrm{H}^{\text {down }}$ on Pt(111)). This nicely demonstrates a "universal" interaction between water and noble metal surfaces featuring a net polarization (or charge-transfer) from water to the surface, i.e., the surface becomes more negatively charged and behaves as a more reductive system compared to vacuum. Note, however, that the arrangements with the lowest surface energy $(\sqrt{39}$ and $\sqrt{37}$ ) feature a $\Delta \Phi$ close to zero and thus also the smallest surface dipole moments.

In summary, both the energetic and the electronic structure analysis support the idea that the lowest energy arrangement of water on noble metal surfaces might resemble the $\sqrt{39}$ structure, i.e., densely packed, but containing various relative orientations of the water molecules.

\section{Energy Decomposition Analysis}

\section{Water-Metal Interaction}

The first, fundamental, question addressed herein is how the interaction of water with a given metal surface depends on the arrangement of the water molecules and on the nature of the metal surface. This question is, furthermore, of importance when aiming at the development of a second generation force field, improving over the existing ones that are fitted to monomer interaction energies, i.e., missing all many body terms. Therefore, we start by analyzing the interaction of the preformed adlayers with the metallic surface, i.e. $\Delta E_{\text {int }}^{\text {surf-layer }}$, and each of its components, defined in analogy to Eq. 8. This means that the deformation energy is excluded, while the water molecules interact with each other freely, i.e., the water-water charge-transfer associated with the hydrogen bonds is present at all stages of the analysis. As a consequence, the water-water CT does not directly contribute to the studied energy difference: the "frozen" term, $\Delta E_{\text {frozen }}^{\text {surf-layer }}$, solely accounts for the electrostatic, steric and dispersion interaction between the adlayer and the metal surface. The polarization term $\Delta E_{\text {pol }}^{\text {surflayer }}$ is mainly composed of the polarization of the metallic surface 


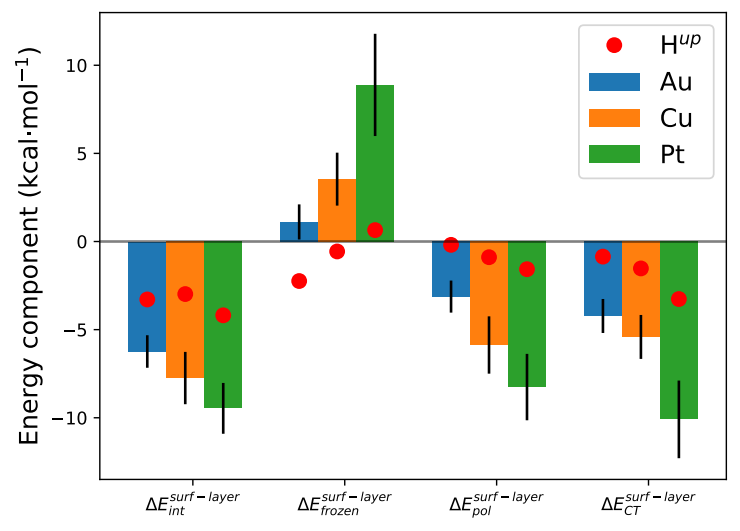

FIG. 4: Average (per water molecule) frozen, polarization, charge-transfer and interaction energy for oligomer and ice adlayer interaction energies with metal surfaces. The error bar gives the standard deviation among all the 27 considered systems. Red dots give the specific values for the $\mathrm{H}^{\text {up }}$ layers.

and the adlayer, but also contains a response of the water-water interaction due to this polarization. Finally, the charge-transfer contribution $\Delta E_{C T}^{\text {surf-layer }}$ captures the charge-transfer between the metal surface and the adlayers and its repercussions on the water-water interaction. To simplify the discussion, we will only discuss the case of three metals: $\mathrm{Pt}, \mathrm{Cu}$ and $\mathrm{Au}$. Indeed, the corresponding values for Pd resemble Pt very closely and the same is true for the couple $\mathrm{Ag}$ and $\mathrm{Au}$. On the other hand, we enrich the discussion by including oligomeric clusters on the surface, in order to deduce more general trends than just observation of the five ice adlayers.

Fig. 4 reports average energies per water molecule for the interaction energy and its components. As expected based on the single molecule adsorption ${ }^{25}$, the average interaction energy is largest for $\mathrm{Pt}(-9.5 \mathrm{kcal} / \mathrm{mol})$ and smallest for $\mathrm{Au}$ $(-6.2 \mathrm{kcal} / \mathrm{mol})$. Note, that this is less than the single molecule adsorption $(-10.6$ and $-7.5 \mathrm{kcal} / \mathrm{mol})$, indicating that the net effect of high coverage is slightly repulsive.

When moving to the components, we can first note a general trend for all components to be, in absolute value, more important for $\mathrm{Pt}$ than for $\mathrm{Cu}$, than for $\mathrm{Au}$. For instance, the steric repulsion, at the origin of the positive sign for $\Delta E_{\text {frozen }}^{\text {surf-layer }}$ is highest for Pt and almost zero for Au. This can be traced back to "geometrical" reasons, with a mean distance $\mathrm{Au}-\mathrm{O}$ of 3.20 vs. Pt-O of 2.98 , which is a consequence of the overall stronger adsorption on $\mathrm{Pt}$, which leads to shorter internuclear distances. The origin of this strong difference in geometry, and thus steric repulsion, is mostly found in $\Delta E_{C T}^{\text {surf-layer }}$, which is more than twice for Pt compared to $\mathrm{Au}(-10.1 \mathrm{vs}-4.2 \mathrm{kcal} / \mathrm{mol})$. The same proportion applies to the polarization energy, but overall, $\Delta E_{\text {pol }}^{\text {surf-layer }}$ is less stabilizing than $\Delta E_{C T}^{\text {surf-layer }}$, except for $\mathrm{Cu}$, where they average to -5.9 and $-5.4 \mathrm{kcal} / \mathrm{mol}$, respectively.

To give an illustration of the spread of the individual com-

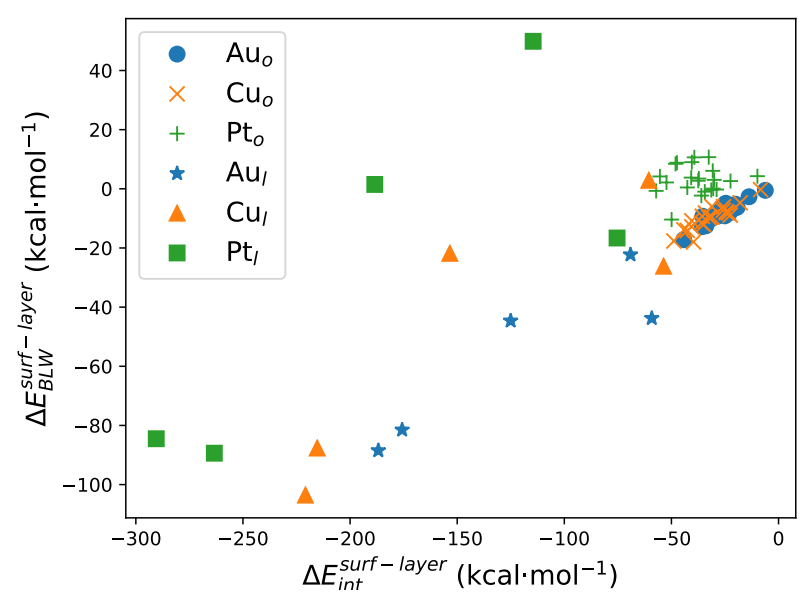

FIG. 5: The interaction of the water subsystem with the metal surface at the BLW level, $\Delta E_{B L W}^{\text {surf-layer }}$, is plotted against the corresponding total interaction energy, $\Delta E_{\text {int }}^{\text {surf-layer }}$. The oligomers (o) and adlayers (1) are given by separated symbols. The (l) point most left and right corresponds to $\sqrt{39}$ and $\mathrm{H}^{\mathrm{up}}$, respectively.

ponents and the profound difference between $\mathrm{H}^{\text {up }}$ compared to the other ice adlayers, let us discuss them at the example of $\operatorname{Pt}(111)$, even though the observations and conclusions for the other metal surfaces would barely differ. First, many water molecules in the ice adlayers are not adsorbed in the optimal single molecule geometry. This contributes to a lowering of the repulsion $\left(\Delta E_{\text {frozen }}^{\text {surf-layer }}\right)$ for the ice adlayers compared to the average $(4.6 \mathrm{vs} .8 .9 \mathrm{kcal} / \mathrm{mol})$. For $\mathrm{H}^{\text {up }}$ this repulsion is even only $0.6 \mathrm{kcal} / \mathrm{mol}$, illustrating the little steric hindrance between the ice-layer and the metal surface. The polarization energy is similarly small for $\mathrm{H}^{\text {up }}(-1.6 \mathrm{kcal} / \mathrm{mol})$ while the two $\mathrm{H}^{\text {down }}$ adlayers feature $\Delta E_{\text {frozen }}^{\text {surf-layer }} \approx-5 \mathrm{kcal} / \mathrm{mol} . \Delta E_{\text {frozen }}^{\text {surf-layer }}$ reaches even $\sim-8 \mathrm{kcal} / \mathrm{mol}$ for the more complex $\sqrt{37}$ and $\sqrt{39}$ structures, a value that compares well to an average of $-8.3 \mathrm{kcal} / \mathrm{mol}$ for all 27 systems considered. The situation for the charge-transfer, $\Delta E_{C T}^{\text {surf-layer }}$, is close to the observations for $\Delta E_{\text {frozen }}^{\text {surf-layer }}$, i.e., $\mathrm{H}^{\text {up }}$ only marginally benefits from $\mathrm{CT}\left(\Delta E_{\text {frozen }}^{\text {surf }}\right.$-layer $=-3.3$ $\mathrm{kcal} / \mathrm{mol}$ ), while the other structures are, with $-7.8 \mathrm{kcal} / \mathrm{mol}$ somewhat shy of the average $\Delta E_{C T}^{\text {surf-layer }}$ of $-10.1 \mathrm{kcal} / \mathrm{mol}$. These observations demonstrate that $\mathrm{H}^{\text {up }}$ behaves differently compared to the other adlayers. However, most of the other ice adlayers, and in particular the $\sqrt{37}$ and $\sqrt{39}$ structures which are the most stable ones, are closely related adsorption patterns that can be mimicked via oligomers. The oligomers have the advantage of offering access to a larger diversity. Furthermore, since they are just molecular clusters on the surface, they can relax and accommodate more easily the various lattice constants, in contrast with the periodic addlayers that need to be stretched or compressed to fit into the unit cell.

From the perspective of designing a force field, the most important question at this point is if the charge-transfer energy 


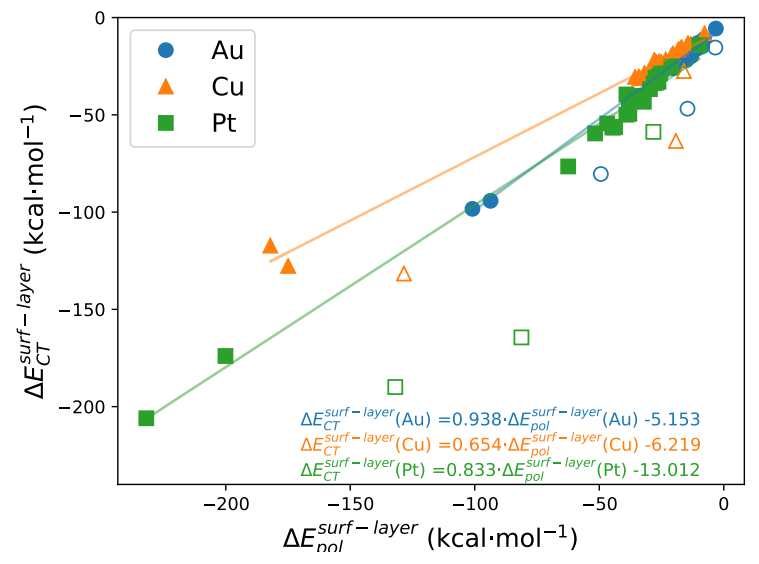

FIG. 6: Correlation of $\Delta E_{C T}^{\text {surf-layer }}$ with $\Delta E_{\text {pol }}^{\text {surf-layer }}$. The empty symbols correspond to the three outlier adlayers $\left(\mathrm{H}^{\text {up }}\right.$, $\mathrm{H}^{\text {down }}$ and chain- $\mathrm{H}^{\text {down }}$ ) which are excluded from the correlation.

between the ice-like layer and the metallic surface is indeed required. Hence, Fig. 5 reports the interaction energy of the water subsystem with the metal surface when charge-transfer is neglected $\left(\Delta E_{B L W}^{\text {surf-layer }}\right)$ as a function of the total interaction energy $\left(\Delta E_{\text {int }}^{\text {sur-layer }}\right)$. To better distinguish the behavior of the oligomers (o) and the complete ice adlayers (1), the two groups are depicted with different symbols, but using the same color. For the oligomers (o) Fig. 5 it is evident that for Pt (green) there is no relation between the BLW (polarization-only) and the total interaction energy. However, for $\mathrm{Cu}$ (orange) and $\mathrm{Au}$ (blue), where the role of CT is less important, there is a good correlation between the two quantities, suggesting that relative adsorption energies could already be estimated at the BLW level. The BLW computations could benefit from a significant speedup due to its (near) linear scaling, dramatically reducing the computational cost of sampling phase space at the metal/liquid interface. When considering the ice adlayers (1) we first see a rough correlation for all three metals which supports the suggestion that CT might not be necessary for relative energies at the metal/liquid interface. At a closer look, the value for $\mathrm{H}^{\text {up }}$ point (rightmost points of the (l) series) does not fit in the correlation for any of the metals. Apparently, $\mathrm{H}^{\text {up }}$ has a non-typical behavior, meaning its properties are significantly different from other water arrangements on noble metal surfaces. We, therefore, advise against its use in practical applications as a model for the water/metal interface.

Even if in the absence of CT a good correlation with $\Delta E_{\text {int }}^{\text {surf-layer }}$ can be obtained, in absolute terms it cannot be neglected: $\Delta E_{\text {int }}^{\text {surf-layer }}$ in the absence of CT, i.e., $\Delta E_{B L W}^{\text {surf-layer }}$, is not even stabilizing for Pt and only mildly so for $\mathrm{Cu}$ and $\mathrm{Au}$ (see Fig. 5). This demonstrates that $\Delta E_{C T}^{\text {surf-layer }}$ is a significant term over all the metals and most important on Pt. Platinum is known to be more oxophilic than $\mathrm{Au}$ and $\mathrm{Cu}$, which is also seen in the water monomer binding energy, which is -10.6 for $\mathrm{Pt}(111)$ vs -8.5 and $-7.5 \mathrm{kcal} / \mathrm{mol}$ for $\mathrm{Cu}$ and $\mathrm{Au}$, respectively. This oxophilicity can explain the importance of CT over $\operatorname{Pt}(111)$. Strikingly, with the exception of the $\mathrm{H}^{\mathrm{up}}$, $\mathrm{H}^{\text {down }}$ and chain- $\mathrm{H}^{\text {down }}$ adlayers, $\Delta E_{C T}^{\text {surf-layer }}$ can be estimated from $\Delta E_{\text {pol }}^{\text {surf-layer }}$ (see Fig. 6). The slope of $\Delta E_{C T}^{\text {surf-layer }}$ vs $\Delta E_{\text {pol }}^{\text {surflayer }}$ only slightly depends on the metal when excluding the "outliers", which are $\mathrm{H}^{\mathrm{up}}, \mathrm{H}^{\text {down }}$ and chain- $\mathrm{H}^{\text {down }}$. The slope is close to unity for $\mathrm{Pt}$ and $\mathrm{Au}$, whereas it is only 0.7 for $\mathrm{Cu}$. However, for the latter the slope rises to 0.9 when excluding all ice adlayers, revealing once again the impact of the lattice mismatch. Hence, if an accurate prediction of the metal/water polarization energy could be found via an empirical force field, the corresponding charge-transfer term could be estimated without a detailed physical model. This possibility opens encouraging perspectives for the next generation of water/metal force fields.

\section{Adsorption energy of water at noble metal surfaces}

Having established that the interaction energy between an ice adlayer, or just a water oligomer, and a noble metal surface can be expressed in terms of the frozen energy and a scaled polarization energy, we now tackle the more general question of the total adsorption energy on metal surface. $\Delta E_{a d s}$, (Eq. 2) accounts for all the many-body interaction terms, i.e., the water-water many-body interactions that are already present in the absence of a metal surface, ${ }^{54}$ the water-metal manybody interactions at the interface and, moreover, the change of the water-water interaction due to the presence of the metal surface.

To settle the stage, Fig. 7 represents the same kind of analysis for $\Delta E_{a d s}$, as Fig. 4 does for $\Delta E_{\text {int }}^{\text {surf-layer }}$, i.e., the different interaction energy components per water molecule for each metal. The first, general, comment is that the two Figures look quite similar, with the same increase in absolute value of all terms when going from $\mathrm{Au}$ to $\mathrm{Cu}$ and then to $\mathrm{Pt}$. The additional energy contribution, $\Delta E_{\text {deform }}$, turns out to be of minor importance overall $(<1 \mathrm{kcal} / \mathrm{mol})$. Even for $\mathrm{Cu}(111)$ the deformation energy is not larger than for $\mathrm{Pt}$, despite the more important lattice missmatch. This can be traced back to the relative rigidity of the water molecules compared to the softer hydrogen bond interactions between them. Hence, while the monomer geometry do not respond much to the unit cell, it is the assembly into an adlayer that has to adapt upon adsorption.

On average, $\Delta E_{a d s}$ only differs by $\sim 2 \mathrm{kcal} / \mathrm{mol}$ per water molecule between $\mathrm{Au}$ (weakest adsorption) and $\mathrm{Pt}$ (strongest adsorption), even though the magnitude of the major adsorption energy components differ by at least a factor of two. In order to uncover if this similarity is only true on average or if it due to a more "universal" similarity of the interaction of water with any of the noble metal surface, Fig. 8 reports the correlation of adsorption energies on $\mathrm{Pt}(111)$ and $\mathrm{Cu}(111)$ with the more physisorption-like adsorption on $\mathrm{Au}(111)$. Due to the large absolute difference between the adsorption energies of oligomers (up to $-90 \mathrm{kcal} / \mathrm{mol}$ ) and ice adlayers (up to $380 \mathrm{kcal} / \mathrm{mol}$ ), the two families of systems are separated. The 


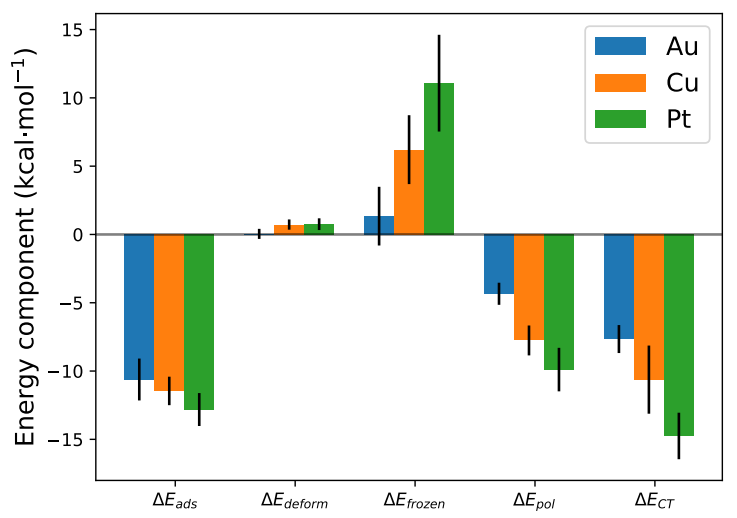

FIG. 7: Average (per water molecule) deformation, frozen, polarization, charge-transfer and adsorption energy for oligomer and ice adlayer adsorption energies with metal surfaces. The error bar gives the standard deviation among all the 27 considered systems.

oligomers (Fig. 8a) have slope close to unity and the intercept reflects the stronger adsorption of a single water molecule on $\mathrm{Cu}$ and $\mathrm{Pt}$ compared to $\mathrm{Au}(111)$. For the ice adlayers (Fig. $8 b), \mathrm{Cu}(111)$ is nearly indistinguishable from $\mathrm{Au}(111)$. The combination of the two figures clearly shows that even though water oligomers are more strongly bound on $\mathrm{Cu}(111)$ than on $\mathrm{Au}(111)$, the lattice-missmatch affects the water adlayers significantly. $\Delta E_{a d s}$ for ice adlayers on $\mathrm{Pt}(111)$ is, with a slope of 1.24 against $\mathrm{Au}(111)$, stronger and indicative of additional stabilization on $\mathrm{Pt}(111)$ compared to the other noble metals compared to the oligomers. This stabilization is presumably due to a combination of stronger chemisorption and a wellmatching metal lattice.

Despite this seemingly simple distinction between adlayers and oligomers when analyzing the differences between metals, the individual components offer a complementary insight. In Fig. 9 we trace $\Delta E_{C T}$ as a function of $\Delta E_{p o l}$ for the three metal surface. When excluding the three "exceptional" and energetically less stable ice adlayers $\left(\mathrm{H}^{\text {up }}, \mathrm{H}^{\text {down }}\right.$ and chain$\mathrm{H}^{\text {down }}$ ), an excellent correlation is obtained with slopes of about 1.5 for all three metals. This slope is higher compared to the near unity slope from Fig. 6, where only the interaction between the adlayer and the metal surface was analyzed. The origin of the difference is two-fold: first and foremost, the water-water interaction, which is directly present in the scaling of Fig. 9, features a comparably stronger CT component with respect to the polarization energy. Second, Fig. 9 also contains the full cooperativity between water-water and water-metal interactions, which are quantified in more details in the next subsection. The linear correlation of Fig. 9 means that $\Delta E_{p o l}$ is sufficient to retrieve the complex many-body physics of $\Delta E_{C T}$, even including the water-water interaction. Therefore, modelling the polarization energy in the absence of chargetransfer should be enough to capture the essential features for the full adsorption energies $\Delta E_{a d s}$.

\section{Non-Additivity and Cooperativity of Water-Water-Metal Many-Body Interactions}

Operationally, modification of the water-water interaction at the metal interface cannot be distinguished from the modification of the water-metal interaction due to the presence of co-adsorbed water molecules. We first quantify and compare the non-additivity of the interaction energy $\left(\Delta E_{i n t}^{\text {NonAdd }}=\right.$ $\left.\Delta E_{\text {frozen }}^{\text {NonAdd }}+\Delta E_{\text {pol }}^{\text {NonAdd }}+\Delta E_{C T}^{\text {NonAdd }}\right)$ for oligomers on $\operatorname{Pt}(111)$ and $\mathrm{Au}(111)$. The non-additivity (Eq. 10) measures the difference in the interaction energy between the sum of single water molecules interacting with the surface and the assembly of all water molecules interacting with the metal surface.

For the oligomers, $\Delta E_{\text {int }}^{\text {NonAdd }}$ contributes to more than $30 \%$ to the total interaction energy. In other words, the nonadditivity is significant for a quantitative understanding of the interactions at the metal/water interface. Fig. S5 demonstrates, however, that $\Delta E_{\text {int }}^{\text {NonAdd }}$ correlates very well $\left(R^{2}=0.93\right.$ and slope of almost unity) between the two extreme metals, $\mathrm{Au}$ (weak adsorption) and $\mathrm{Pt}$ (strong adsorption), suggesting that it is a "universal" quantity. The components of $\Delta E_{\text {int }}^{\text {NonAdd }}$ do not all behave the same: The repulsive frozen terms is very weakly correlated $\left(R^{2} \approx 0.5\right)$, but noticeably smaller for Au than for $\mathrm{Pt}$ (roughly one third). This is to be expected since the water molecules are further away from the surface on $\mathrm{Au}$ than on $\mathrm{Pt}$. Oppositely, the non-additive charge-transfer and polarization energy are quite well correlated between Pt and Au and bring about the correlation between the metals.

Having established the "universal" character of the nonadditivity interaction, we now focus on the case of $\operatorname{Pt}(111)$ to obtain a geometric understanding of its origin. Since the structures are essentially two-dimensional, we do not simply determine the coordination number, ${ }^{55}$ but perform a directional analysis: in each structure, the $\mathrm{H}$-bond $(\mathrm{H} \cdots \mathrm{O}$ distance below $2.5 \AA$ ) acceptors are identified. Then, they are classified according to the $\mathrm{Pt}-\mathrm{O}$ distance $(<3.0 \AA$ for chemisorbed water molecules, $>3.0 \AA$ for physisorbed molecules). The $\theta$ and $\phi$ rotational angles, which describe the rotation of the water dipole moment with respect to the surface normal and the rotation of the molecular plane around the dipole moment, respectively, provide natural, additional parameters (see Fig. $\mathrm{S} 4$ for a graphical definition). In order to simplify the analysis, the two-dimensional space spanned by $\theta$ and $\phi$ is divided into 9 rectangles for the physisorbed water molecules. The limits of these rectangles are optimized to find the optimal linear model reproducing the non-additivity for all 27 structures, i.e., including the ice adlayers. For the chemisorbed molecules, only two combinations are necessary (see Table S1). The root mean square error of this linear regression amounts to $1.37 \mathrm{kcal} / \mathrm{mol}$ (see Fig. S6), demonstrating the good predictive power of this simple model. The advantage of the linear model is that we also gain insight into geometrical arrangements that are responsible for the non-additivity. The corresponding energy coefficients for chemisorbed molecules (see Table S1) indicate that the typical adsorption minimum of a single water molecule $\left(\theta \approx 80^{\circ}, \phi \approx 0^{\circ}, \mathrm{Pt}-\mathrm{O} \approx 2.5 \AA\right)$ is the worst $\mathrm{H}$-bond acceptor $\left(\Delta E_{\text {int }}^{\text {acceptor,NonAdd }}=-3.0 \mathrm{kcal} / \mathrm{mol}\right)$, i.e., does not contribute significantly to the non-additivity. This is compatible with the 


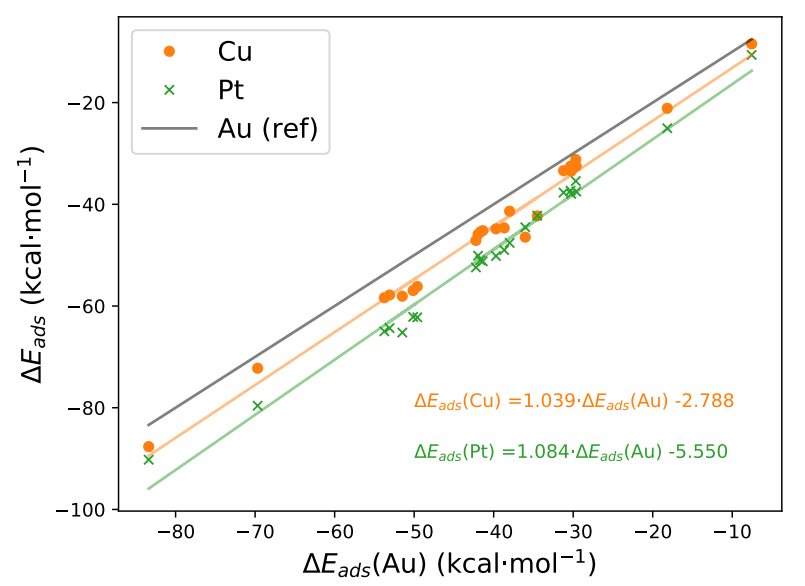

(a) Oligomers

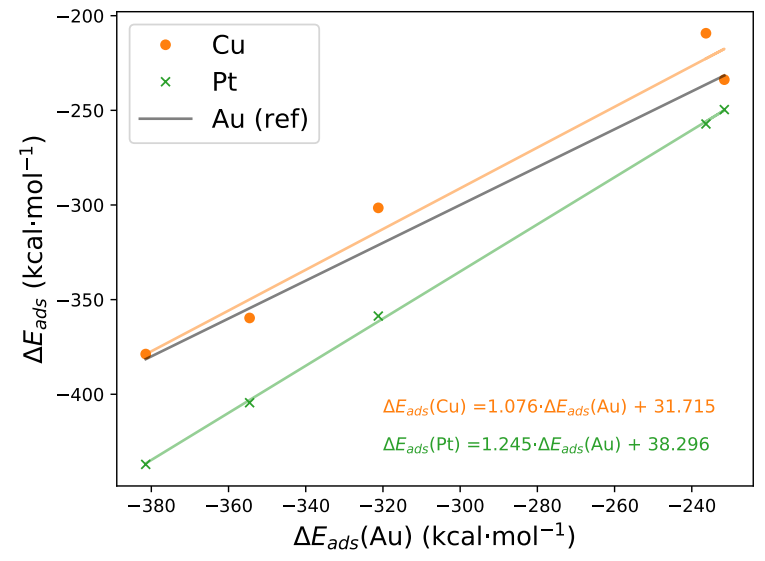

(b) Ice adlayers

FIG. 8: The adsorption energy for oligomers (a) and ice adlayers (b) on $\mathrm{Cu}(111)$ and $\mathrm{Pt}(111)$ is plotted against the adsorption energy on $\mathrm{Au}(111)$.

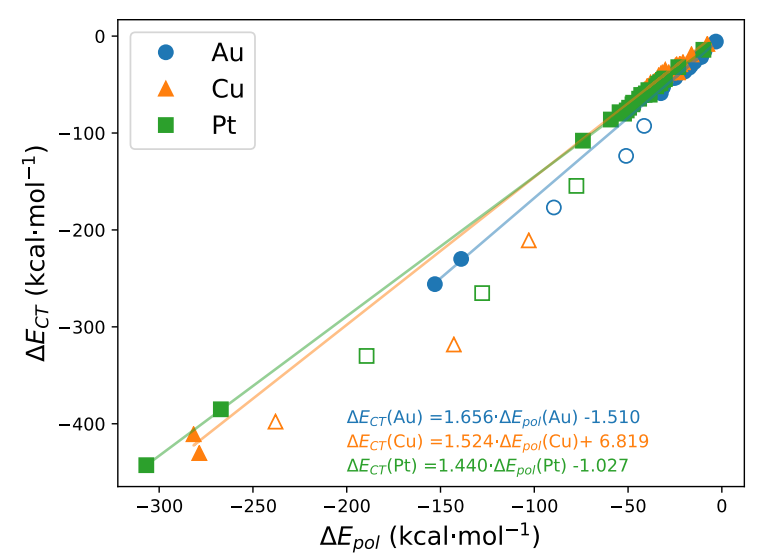

FIG. 9: The charge-transfer $\Delta E_{C T}$ is correlated to $\Delta E_{p o l}$ for the adsorption energy. The empty symbols correspond to the three outlier adlayers $\left(\mathrm{H}^{\mathrm{up}}, \mathrm{H}^{\text {down }}\right.$ and chain- $\left.\mathrm{H}^{\text {down }}\right)$ which are excluded from the correlation.

observation that the oxygen atom is already interacting with the metal surface via its lone-pairs. Therefore, its electrons are less available to interact with a third hydrogen atom. In contrast, the typical building block of the ice-like layers, where the chemisorbed water molecule is tilted so that the hydrogens are pointing away from the surface $\left(\theta \approx 50^{\circ}, \phi \approx 0, \mathrm{Pt}-\mathrm{O} \approx 2.5 \AA\right)$ is a better $\mathrm{H}$-bond acceptor $(-5.4 \mathrm{kcal} / \mathrm{mol})$. The best $\mathrm{H}$-bond acceptors are, however, not chemisorbed to the surface but physisorbed $(\mathrm{Pt}-\mathrm{O}>3.0)$, and present all small $\phi$ values $\left(<70^{\circ}\right)$, meaning that the two hydrogens are at somewhat similar distances from the surface. The very best region $(-10.4 \mathrm{kcal} / \mathrm{mol})$ is found for $\theta>120^{\circ}$, which corresponds to two hydrogens pointing toward the surface, but with a lone pair of the oxygen atom pointing in the direction of the surface, and thus, towards

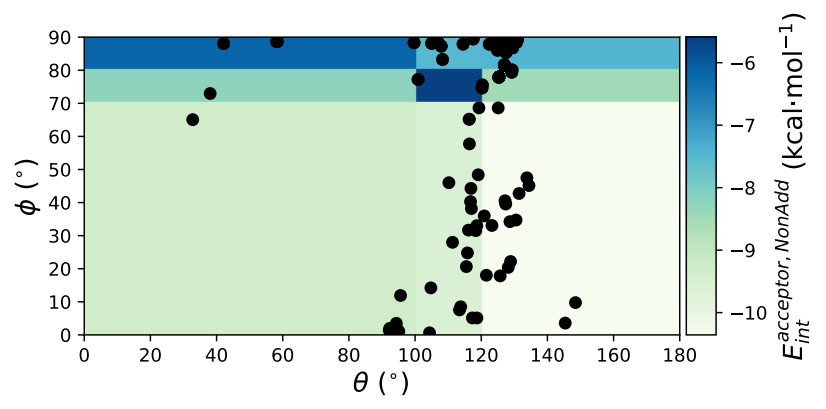

FIG. 10: Representation of the non-additive energy contribution of physisorbed $\mathrm{H}$-bond acceptors as a function of their $\theta$ and $\phi$ characteristics. Dots indicate the observed points. The data is also available in Table S1

a potential H-bond donnor. A more in-depth study on model systems would be necessary in order to deduce clearer trends and adapted functional forms to reproduce these trends in an empirical force field.

Now that we understand the geometrical origin of the non-additivity, we unravel its origin in terms of contributing components. Overall, $\Delta E_{\text {frozen }}^{\text {NonAd }}$ is destabilizing while both the polarization and charge-transfer energies are stabilizing the adlayer and are responsible for $\sim 40 \%$ and $60 \%$ of $\Delta E_{\text {int }}^{\text {NonAdd }}-\Delta E_{\text {frozen }}^{\text {NonAdd }}$ (i.e. the stabilizing component), respectively and are highly correlated with each other $\left(R^{2}=0.98\right)$. The major exception to this trend is, again, the $\mathrm{H}^{\mathrm{up}}$ layer for which the frozen interaction is attractive, but the polarization 
and charge transfer provide less additional stability compared to the average.

\section{The synergy between water-water charge transfer and water-metal charge transfer}

The synergistic energy can be defined as $\Delta E_{C T}^{s y n}=$ $\Delta E_{C T}^{\text {NonAdd }}-E_{C T}\left(\bigcup_{i} W_{i}\right)=\Delta E_{C T}-\sum_{i}^{n} \Delta E_{C T}\left(W_{i}\right.$, surf $)-$ $E_{C T}\left(\bigcup_{i} W_{i}\right)$, which represents the CT-associated energetic difference between, in one hand, the overall CT and, and in the other hand, the sum of each individual charge transfer between a single water molecule and the surface plus the charge transfer within the isolated water layer. This synergy is therefore positive (destabilizing) if there is a competition between these charge transfers and negative for (stabilizing) cooperativity. Overall, cooperativity is found with $\Delta E_{C T}^{s y n}=-1.37 \mathrm{kcal} / \mathrm{mol}$ per water molecule on average for Pt and $-0.57 \mathrm{kcal} / \mathrm{mol}$ for Au. For $\mathrm{H}^{\text {up }}$, however, $\Delta E_{C T}^{s y n}$ is positive for both $\mathrm{Pt}$ and $\mathrm{Au}$ (1.1 and $0.5 \mathrm{kcal} / \mathrm{mol}$ per water molecule, respectively). On the opposite, the most stable layers $(\sqrt{37}, \sqrt{39})$ are the ones where this cooperation is higher, with also small net dipole moments and large contributions due to polarization.

\section{CONCLUSION}

The detailed analysis of the electronic and geometrical characteristics of 27 diverse water arrangements (from monomer to ice adlayers) over the (111) surface of five noble metals $(\mathrm{Cu}, \mathrm{Ag}, \mathrm{Au}, \mathrm{Pd}$ and $\mathrm{Pt})$ has allowed to identify trends and key factors for the stability of water arrangements on metallic surfaces. We identified dense-packed layers to be the most stable structures, like the $\sqrt{39} \times \sqrt{39}$ unit cell for $\mathrm{Ag}, \mathrm{Au}, \mathrm{Pd}$ and $\mathrm{Pt}$ and the $\sqrt{37} \times \sqrt{37}$ unit-cell for $\mathrm{Cu}$. This stability was found to correspond to the smallest change of workfunction upon adsorption. The $\mathrm{H}^{\text {down }}$ and $\mathrm{H}^{\text {up }}$ structures, which are often cited as model structure for water/metal interfaces, are less stable and lead to workfunction changes up to $3 \mathrm{eV}$.

The energy decomposition analysis, relying on the block localized wave function (BLW), shows that the charge-transfer from water to the surface is overall nearly independent from the charge-transfer between the water molecules, the latter being key for the H-bonding. $\Delta E_{C T}$, which is the computationally most costly term, is found to be linearly correlated to $\Delta E_{p o l}$. Hence, it can be predicted at minimal cost. As a consequence, the polarization energy can be seen as the most important contribution to the adsorption energy.

Remarkably, the polarization energy provides about $40 \%$ additional stability at the interface compared to the single water molecule adsorption, and displays a strong correlation with its charge-transfer counterpart $\left(R^{2}=0.99\right.$ on $\left.\mathrm{Pt}(111)\right)$. This is good news for force field developments, where models for the polarization energy could be included to capture the manybody effects. Alternatively, this cooperativity can be largely reproduced by an additive model based on the geometric pa- rameters of the H-bond acceptor molecule. In summary, our investigation highlights the closely related physics that governs the various noble-metal - water interaction and suggests that polarization energies should be enough to retrieve most of the complex many-body interactions at the metal/water interface.

\section{ACKNOWLEDGMENTS}

The authors thank the SYSPROD project and AXELERA Pôle de Compétitivité for financial support (PSMN Data Center). We are grateful to A. Michaelides for providing some of the ice adlayers and to $\mathrm{C}$. Michel for stimulating discussions.

${ }^{1}$ D. L. Doering and T. E. Madey, "The adsorption of water on clean and oxygen-dosed Ru(011)," Surface Science 123, 305-337 (1982).

${ }^{2}$ A. Glebov, A. P. Graham, A. Menzel, and J. P. Toennies, "Orientational ordering of two-dimensional ice on $\operatorname{Pt}(111)$," The Journal of Chemical Physics 106, 9382-9385 (1997).

${ }^{3}$ S. Nie, P. J. Feibelman, N. C. Bartelt, and K. Thürmer, "Pentagons and Heptagons in the First Water Layer on Pt(111)," Phys. Rev. Lett. 105, 026102 (2010).

${ }^{4}$ J. Carrasco, A. Hodgson, and A. Michaelides, "A molecular perspective of water at metal interfaces," Nature Materials 11, 667-674 (2012), number: 8 Publisher: Nature Publishing Group.

${ }^{5}$ H. Ogasawara, B. Brena, D. Nordlund, M. Nyberg, A. Pelmenschikov, L. G. M. Pettersson, and A. Nilsson, "Structure and Bonding of Water on Pt(111)," Phys. Rev. Lett. 89, 276102 (2002).

${ }^{6}$ J. Rossmeisl, J. K. Norskov, C. D. Taylor, M. J. Janik, and M. Neurock, "Calculated Phase Diagrams for the Electrochemical Oxidation and Reduction of Water over Pt(111)," The Journal of Physical Chemistry B 110, 21833-21839 (2006).

${ }^{7}$ S. Schnur and A. Groß, "Properties of metal-water interfaces studied from first principles," New J. Phys. 11, 125003 (2009).

${ }^{8}$ T. Schiros, K. J. Andersson, L. G. M. Pettersson, A. Nilsson, and H. Ogasawara, "Chemical bonding of water to metal surfaces studied with core-level spectroscopies," J. Electron Spectrosc. Relat. Phenom. 177, 85-98 (2010).

${ }^{9}$ J.-S. Filhol and M.-L. Doublet, "An ab initio study of surface electrochemical disproportionation: The case of a water monolayer adsorbed on a $\operatorname{Pd}(111)$ surface," Electrocatalysis 202, 87-97 (2013).

${ }^{10} \mathrm{P}$. Vassilev, R. A. van Santen, and M. T. M. Koper, "Ab initio studies of a water layer at transition metal surfaces,” J. Chem. Phys. 122, 054701 (2005), publisher: American Institute of Physics.

${ }^{11}$ O. Bjorneholm, M. H. Hansen, A. Hodgson, L.-M. Liu, D. T. Limmer, A. Michaelides, P. Pedevilla, J. Rossmeisl, H. Shen, G. Tocci, E. Tyrode, M.-M. Walz, J. Werner, and H. Bluhm, "Water at Interfaces," Chem. Rev. 116, 7698-7726 (2016).

${ }^{12}$ S. K. Desai, V. Pallassana, and M. Neurock, "A periodic density functional theory analysis of the effect of water molecules on deprotonation of acetic acid over pd(111)," J. Phys. Chem. B 105, 9171-9182 (2001).

${ }^{13}$ J.-S. Filhol and M. Neurock, "Elucidation of the Electrochemical Activation of Water over Pd by First Principles," Angew. Chem., Int. Ed. 45, 402-406 (2006).

${ }^{14}$ E. Skulason, G. S. Karlberg, J. Rossmeisl, T. Bligaard, J. Greeley, H. Jonsson, and J. K. Norskov, "Density functional theory calculations for the hydrogen evolution reaction in an electrochemical double layer on the $\mathrm{Pt}(111)$ electrode," Phys. Chem. Chem. Phys. 9, 3241-3250 (2007).

${ }^{15}$ F. Tian, R. Jinnouchi, and A. B. Anderson, "How Potentials of Zero Charge and Potentials for Water Oxidation to $\mathrm{OH}(\mathrm{ads})$ on $\mathrm{Pt}(111)$ Electrodes Vary With Coverage," J. Phys. Chem. C 113, 17484-17492 (2009), publisher: American Chemical Society.

${ }^{16}$ J. Hussain, H. Jónsson, and E. Skúlason, "Calculations of Product Selectivity in Electrochemical CO2 Reduction,” ACS Catal. 8, 5240-5249 (2018).

${ }^{17}$ K. Mathew, V. S. C. Kolluru, S. Mula, S. N. Steinmann, and R. G. Hennig, "Implicit self-consistent electrolyte model in plane-wave density-functional theory," J. Chem. Phys. 151, 234101 (2019).

${ }^{18}$ S. N. Steinmann, P. Sautet, and C. Michel, "Solvation free energies for 
periodic surfaces: comparison of implicit and explicit solvation models," Phys. Chem. Chem. Phys. 18, 31850-31861 (2016).

${ }^{19}$ D. D. Hibbitts, B. T. Loveless, M. Neurock, and E. Iglesia, "Mechanistic Role of Water on the Rate and Selectivity of Fischer-Tropsch Synthesis on Ruthenium Catalysts," Angew. Chem. Int. Ed. 52, 12273-12278 (2013).

${ }^{20}$ B. Schweitzer, S. N. Steinmann, and C. Michel, "Can microsolvation effects be estimated from vacuum computations? A case-study of alcohol decomposition at the H2O/Pt(111) interface," Phys. Chem. Chem. Phys. 21 , 5368-5377 (2019).

${ }^{21}$ S. N. Steinmann, R. Ferreira De Morais, A. W. Gotz, P. Fleurat-Lessard, M. Iannuzzi, P. Sautet, and C. Michel, "Force Field for Water over Pt(111): Development, Assessment, and Comparison," J. Chem. Theory Comput. 14, 3238-3251 (2018).

${ }^{22}$ J. Carrasco, A. Michaelides, and M. Scheffler, "Insight from first principles into the nature of the bonding between water molecules and $4 \mathrm{~d}$ metal surfaces," J. Chem. Phys. 130, 184707 (2009).

${ }^{23}$ A. Michaelides, V. A. Ranea, P. L. de Andres, and D. A. King, "General Model for Water Monomer Adsorption on Close-Packed Transition and Noble Metal Surfaces," Phys. Rev. Lett. 90, 216102 (2003).

${ }^{24}$ T. Schiros, H. Ogasawara, L.-A. Näslund, K. J. Andersson, J. Ren, S. Meng, G. S. Karlberg, M. Odelius, A. Nilsson, and L. G. M. Pettersson, "Cooperativity in Surface Bonding and Hydrogen Bonding of Water and Hydroxyl at Metal Surfaces," J. Phys. Chem. C 114, 10240-10248 (2010).

${ }^{25}$ J. Carrasco, J. Klimeš, and A. Michaelides, "The role of van der Waals forces in water adsorption on metals," J. Chem. Phys. 138, 024708 (2013), publisher: American Institute of Physics.

${ }^{26}$ J. Carrasco, B. Santra, J. Klimeš, and A. Michaelides, "To Wet or Not to Wet? Dispersion Forces Tip the Balance for Water Ice on Metals," Phys. Rev. Lett. 106, 026101 (2011), publisher: American Physical Society.

${ }^{27}$ Y. Mo, J. Gao, and S. D. Peyerimhoff, "Energy decomposition analysis of intermolecular interactions using a block-localized wave function approach," J. Chem. Phys. 112, 5530-5538 (2000).

${ }^{28}$ R. Z. Khaliullin, A. T. Bell, and M. Head-Gordon, "Analysis of charge transfer effects in molecular complexes based on absolutely localized molecular orbitals," J. Chem. Phys. 128, 184112 (2008).

${ }^{29}$ S. N. Steinmann, P. Vogel, Y. Mo, and C. Corminboeuf, "The norbornene mystery revealed," Chem. Commun. 47, 227-229 (2011).

${ }^{30}$ H. Elgabarty, R. Z. Khaliullin, and T. D. Kuhne, "Covalency of hydrogen bonds in liquid water can be probed by proton nuclear magnetic resonance experiments," Nat. Commun. 6 (2015), 10.1038/ncomms9318.

${ }^{31}$ P. H. T. Philipsen and E. J. Baerends, "Role of the Fermi Surface in Adsorbate-Metal Interactions: An Energy Decomposition Analysis," J. Phys. Chem. B 110, 12470-12479 (2006).

${ }^{32}$ L. Pecher, S. Laref, M. Raupach, and R. Tonner, "Ethers on Si(001): A Prime Example for the Common Ground between Surface Science and Molecular Organic Chemistry,” Angew. Chem., Int. Ed. 56, 15150-15154 (2017).

${ }^{33}$ R. Staub, M. Iannuzzi, R. Z. Khaliullin, and S. N. Steinmann, "Energy Decomposition Analysis for Metal Surface? @ sAdsorbate Interactions by Block Localized Wave Functions," Journal of Chemical Theory and Computation 15, 265-275 (2019).

${ }^{34}$ H. Stoll, G. Wagenblast, and H. Preuss, "On the use of local basis sets for localized molecular orbitals," Theor. Chim. Acta 57, 169-178 (1980).

${ }^{35}$ Y. Mo and S. D. Peyerimhoff, "Theoretical analysis of electronic delocalization," J. Chem. Phys. 109, 1687-1697 (1998).

${ }^{36}$ S. N. Steinmann, D. F. Jana, J. I.-C. Wu, P. v. R. Schleyer, Y. Mo, and C. Corminboeuf, "Direct Assessment of Electron Delocalization Using
NMR Chemical Shifts,” Angew. Chem., Int. Ed. 48, 9828-9833 (2009).

${ }^{37}$ O. Demerdash, Y. Mao, T. Liu, M. Head-Gordon, and T. Head-Gordon, "Assessing many-body contributions to intermolecular interactions of the AMOEBA force field using energy decomposition analysis of electronic structure calculations," J. Chem. Phys. 147, 161721 (2017).

${ }^{38}$ R. Z. Khaliullin, J. VandeVondele, and J. Hutter, "Efficient Linear-Scaling Density Functional Theory for Molecular Systems," Journal of Chemical Theory and Computation 9, 4421-4427 (2013).

${ }^{39}$ S. F. Boys and F. Bernardi, "The calculation of small molecular interactions by the differences of separate total energies. Some procedures with reduced errors," Mol. Phys. 19, 553 - 566 (1970).

${ }^{40}$ R. Z. Khaliullin, E. A. Cobar, R. C. Lochan, A. T. Bell, and M. Head-Gordon, "Unravelling the Origin of Intermolecular Interactions Using Absolutely Localized Molecular Orbitals," J. Phys. Chem. A 111, 8753-8765 (2007).

${ }^{41}$ S. N. Steinmann, C. Corminboeuf, W. Wu, and Y. Mo, "DispersionCorrected Energy Decomposition Analysis for Intermolecular Interactions Based on the BLW and dDXDM Methods," J. Phys. Chem. A 115, 54675477 (2011).

${ }^{42} \mathrm{G}$. Kresse and J. Hafner, "Ab initio molecular dynamics for liquid metals," Phys. Rev. B 47, 558 (1993).

${ }^{43}$ G. Kresse and J. Furthmuller, "Efficient iterative schemes for ab initio totalenergy calculations using a plane-wave basis set," Phys. Rev. B 54, 11169 (1996).

${ }^{44}$ J. Klimes, D. R. Bowler, and A. Michaelides, "Chemical accuracy for the van der Waals density functional," J. Phys.: Condens. Matter 22, 022201 (2010).

${ }^{45}$ P. E. Blochl, "Projector augmented-wave method,” Phys. Rev. B 50, 17953 (1994).

${ }^{46}$ G. Kresse and D. Joubert, "From ultrasoft pseudopotentials to the projector augmented-wave method," Phys. Rev. B 59, 1758 (1999).

${ }^{47}$ J. VandeVondele, M. Krack, F. Mohamed, M. Parrinello, T. Chassaing, and J. Hutter, "Quickstep: Fast and accurate density functional calculations using a mixed Gaussian and plane waves approach," Comput. Phys. Commun. 167, $103-128$ (2005).

${ }^{48}$ J. Hutter, M. Iannuzzi, F. Schiffmann, and J. VandeVondele, "cp2k: atomistic simulations of condensed matter systems," WIREs Comput Mol Sci 4, 15-25 (2014).

${ }^{49}$ J. VandeVondele and J. Hutter, "Gaussian basis sets for accurate calculations on molecular systems in gas and condensed phases," J. Chem. Phys. 127, 114105 (2007).

${ }^{50} \mathrm{~S}$. Goedecker, M. Teter, and J. Hutter, "Separable dual-space Gaussian pseudopotentials," Phys. Rev. B 54, 1703-1710 (1996).

${ }^{51}$ J. R. Rumptz and C. T. Campbell, "Adhesion Energies of Solvent Films to Pt(111) and Ni(111) Surfaces by Adsorption Calorimetry," ACS Catal. 11819-11825 (2019).

${ }^{52} \mathrm{~W}$. Tyson and W. Miller, "Surface free energies of solid metals: Estimation from liquid surface tension measurements," Surface Science 62, 267-276 (1977).

${ }^{53}$ J. J. Mortensen, B. Hammer, and J. K. Nørskov, "Alkali Promotion of \$N_2\$ Dissociation over Ru(0001)," Phys. Rev. Lett. 80, 4333-4336 (1998).

${ }^{54}$ S. K. Reddy, S. C. Straight, P. Bajaj, C. Huy Pham, M. Riera, D. R. Moberg, M. A. Morales, C. Knight, A. W. Götz, and F. Paesani, "On the accuracy of the MB-pol many-body potential for water: Interaction energies, vibrational frequencies, and classical thermodynamic and dynamical properties from clusters to liquid water and ice," J. Chem. Phys. 145, 194504 (2016), publisher: American Institute of Physics.

${ }^{55}$ R. Staub and S. N. Steinmann, "Parameter-free coordination numbers for solutions and interfaces," J. Chem. Phys. 152, 024124 (2020). 


\section{Supporting Information:}

\section{Water Adlayers on Noble Metal Surfaces:}

\section{Insights from Energy Decomposition Analysis}

Paul Clabaut, Ruben Staub, Joachim Galiana, Elise Antonetti, and Stephan N. Steinmann*

Univ Lyon, Ecole Normale Supérieure de Lyon, CNRS Université Lyon 1, Laboratoire de Chimie UMR 5182, 46 allée d'Italie, F-69364, LYON, France

E-mail: stephan.steinmann@ens-lyon.fr

\section{Contents}

1 Additional Analysis $\quad$ S-1

1.1 Water-Water interaction $\ldots \ldots \ldots \ldots \ldots$. . . . . . . . . . .

2 Addtional Tables and Figures $\quad$ S-2

$\begin{array}{lr}\text { References } & \text { S-9 }\end{array}$

\section{Additional Analysis}

\subsection{Water-Water interaction}

The water-water interaction as assessed in the absence of the metal surfaces amounts to -4 (Pt) to -5 $(\mathrm{Au}) \mathrm{kcal} / \mathrm{mol}$ per water molecule, indicating that the chemisorption on $\operatorname{Pt}(111)$ imposes stronger 
constraints on the water geometries than the weaker interaction with $\mathrm{Au}(111)$.

In other words, the chemisorption on Pt leads to water layers that adopt less favorable geometries than on $\mathrm{Au}$, with the deformation energy at the single molecule level being negligible. Interestingly, $\mathrm{Cu}$ is, in this respect, closer to the behavior of $\mathrm{Pt}$ than of $\mathrm{Au}$. This is likely a coincidence, with the lattice missmatch on $\mathrm{Cu}(111)$ playing the role of the chemisorption on $\mathrm{Pt}(111)$.

The water-water interaction is, in magnitude, dominated by the $\Delta E_{C T}$, which contributes -4.0 $\mathrm{kcal} / \mathrm{mol}$ per water molecule over $\mathrm{Pt}(111)$, close to the $-4.2 \mathrm{kcal} / \mathrm{mol}$ average total stabilization.

The water-water interaction contributes $-10 \mathrm{kcal} / \mathrm{mol}$ per water molecule, more than twice as much as on average, to the stability of the $H^{\text {up }}$ add-layer. In contrast, the $\sqrt{37}$ and $\sqrt{39}$ structures benefit "only" from a stabilization of about $-6 \mathrm{kcal} / \mathrm{mol}$ per molecule, once again pointing to the fact that the $H^{\text {up }}$ layer is "peculiar" and not a typical case for the interaction of water with metal surfaces.

\section{Addtional Tables and Figures}

Table S1: $E_{a d s}^{\text {acceptor }}\left(\mathrm{kcal} \cdot \mathrm{mol}^{-1}\right)$ for acceptor with a distance to the surface $\mathrm{z}>3.0$

\begin{tabular}{l|ccc}
$\theta\left(^{\circ}\right) / \phi\left(^{\circ}\right)$ & {$[0-100]$} & {$[100-120]$} & {$[120-180]$} \\
\hline$[80-90]$ & -6.2 & -7.5 & -7.4 \\
{$[70-80]$} & -8.2 & -5.6 & -8.5 \\
{$[0-70]$} & -9.3 & -9.6 & -10.4
\end{tabular}

For chemisorbed acceptors (with a distance to the surface $\mathrm{z}<3.0$ ), $E_{\text {ads }}^{\text {acceptor }}=-5.4 \mathrm{kcal} \cdot \mathrm{mol}^{-1}$ if $\theta<60^{\circ}$ and $-3.0 \mathrm{kcal} \cdot \mathrm{mol}^{-1}$ else. 


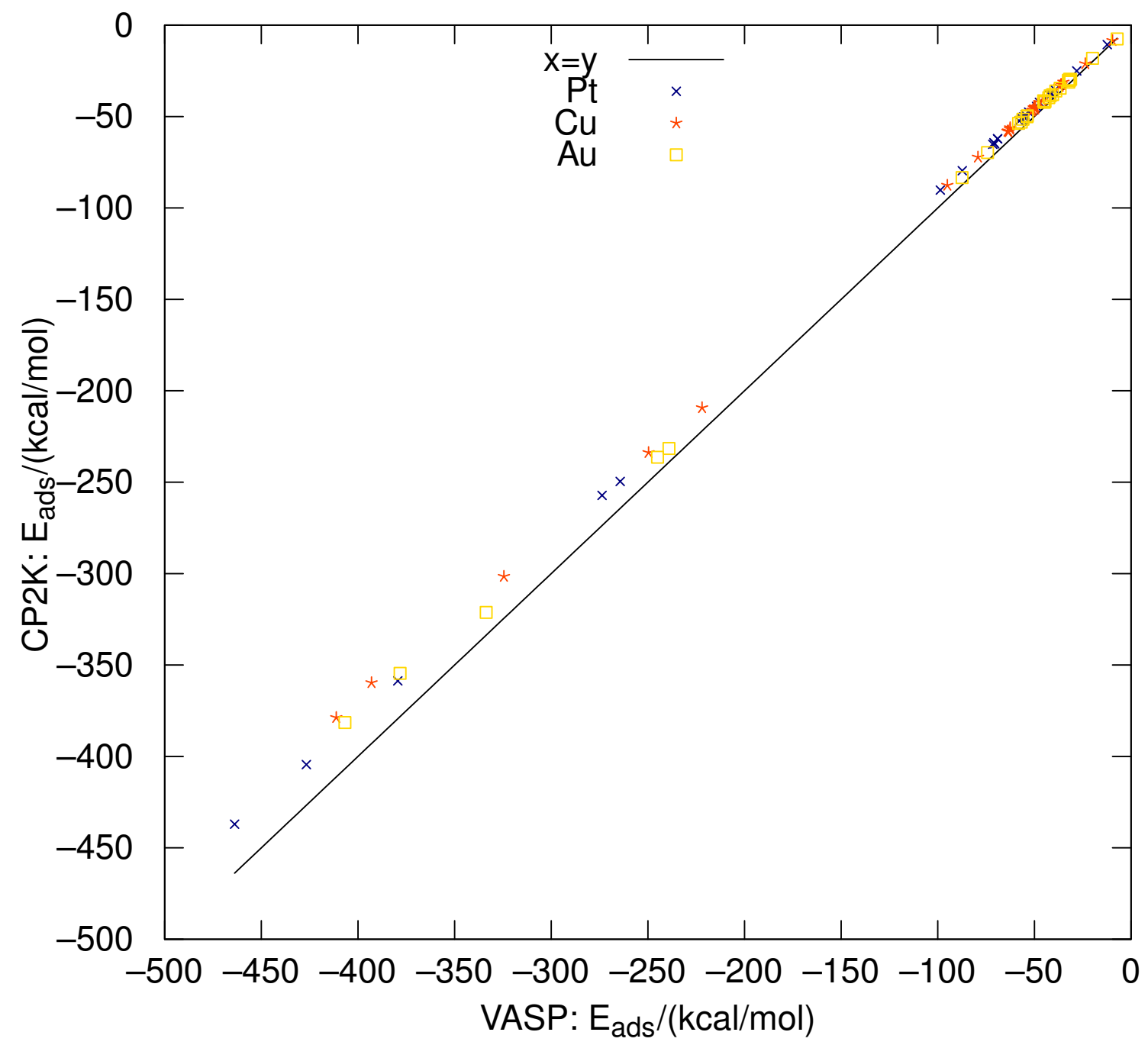

Figure S1: Interaction energies obtained with CP2K plotted against the ones from VASP. The black line correponds to the bisector. 


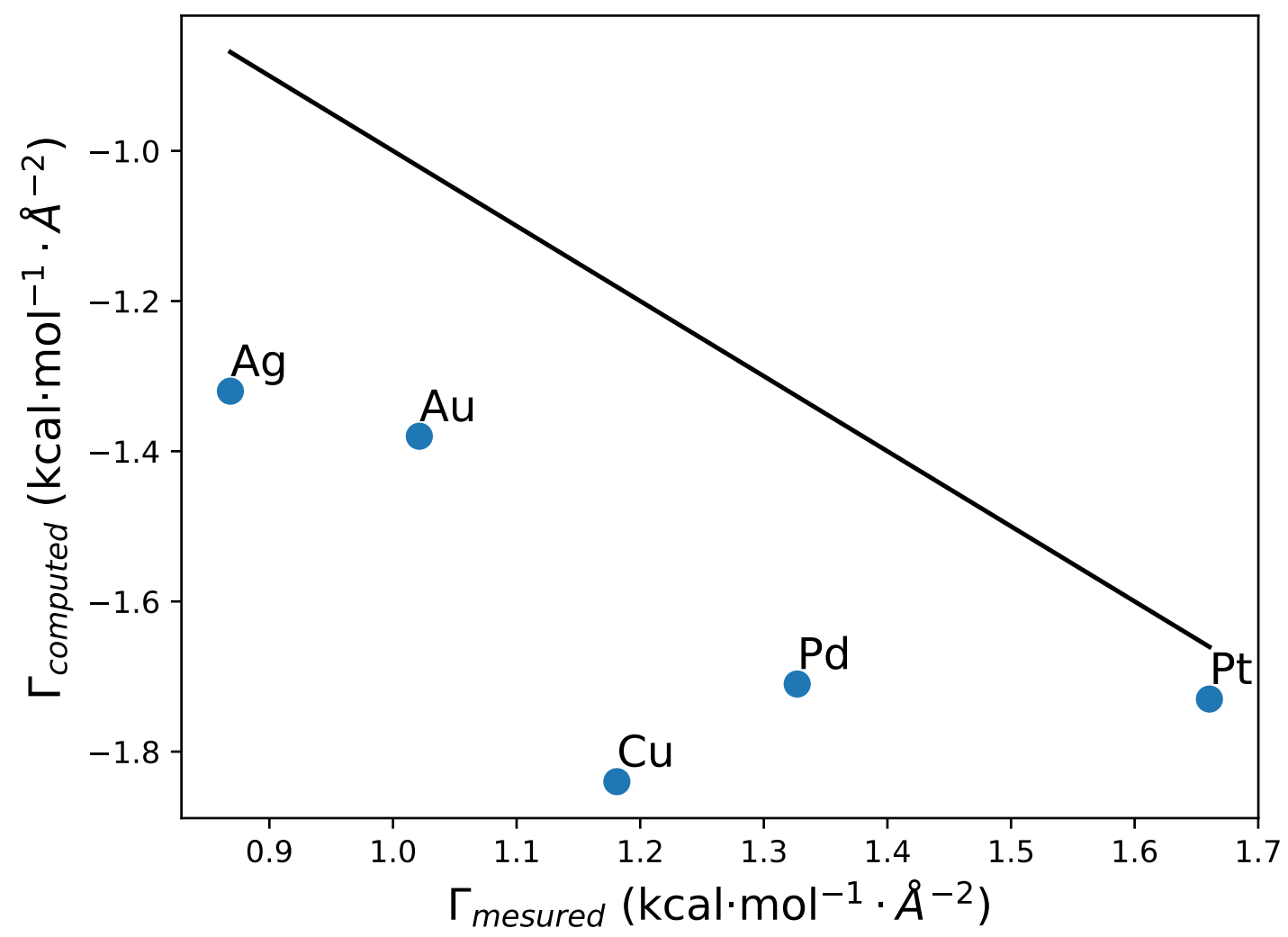

Figure S2: Surface energies in $\mathrm{kcal} / \mathrm{mol} / \AA^{2}$ of the most stable ice-like water layer as a function of the experimental metal/liquid tension (retrieved from ref S1). The black line corresponds to the bisector. 


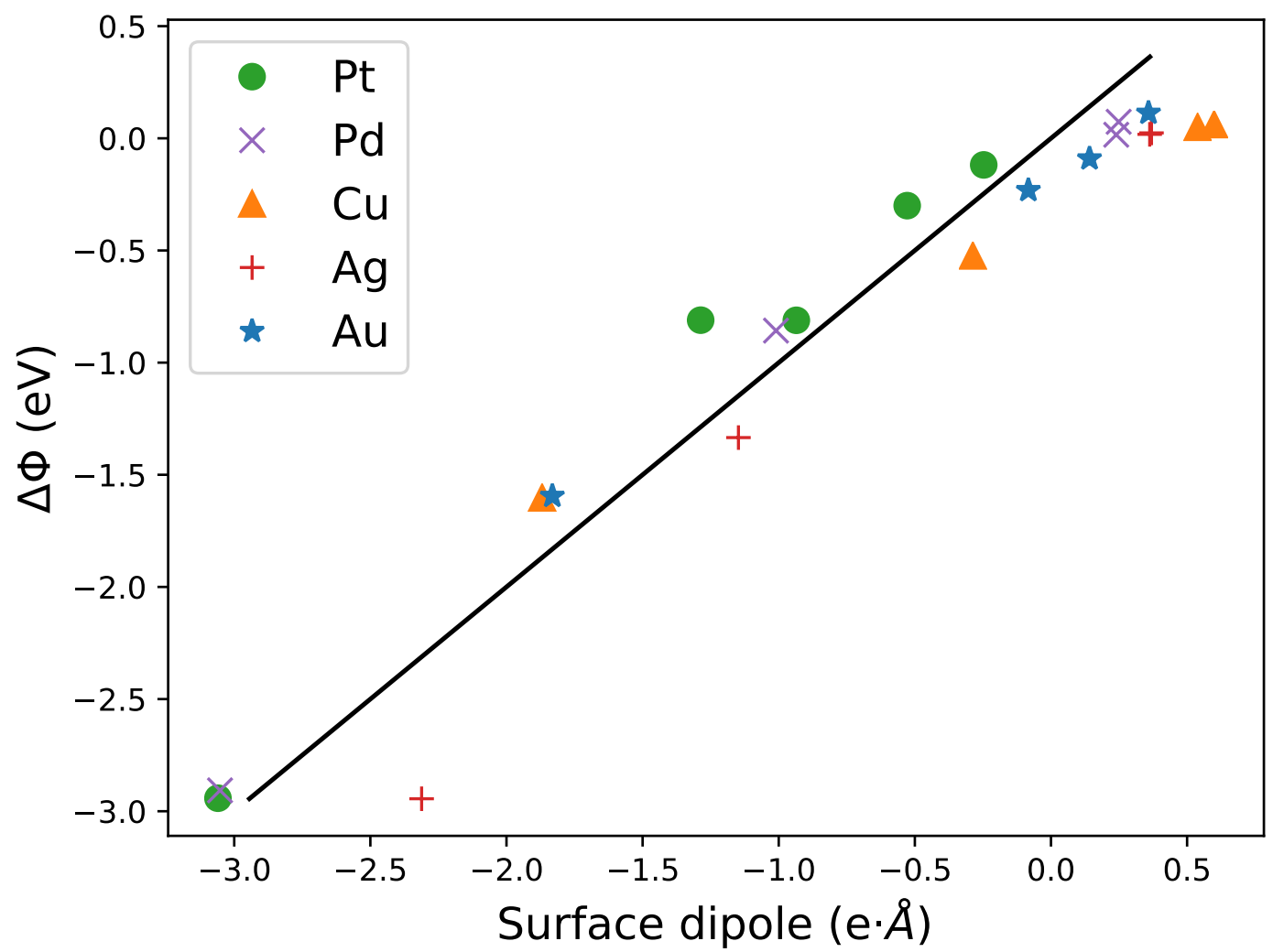

Figure S3: The correlation between the surface dipole moment and the change in workfunction $\Delta \Phi$ upon adsorption of ice add-layers. 


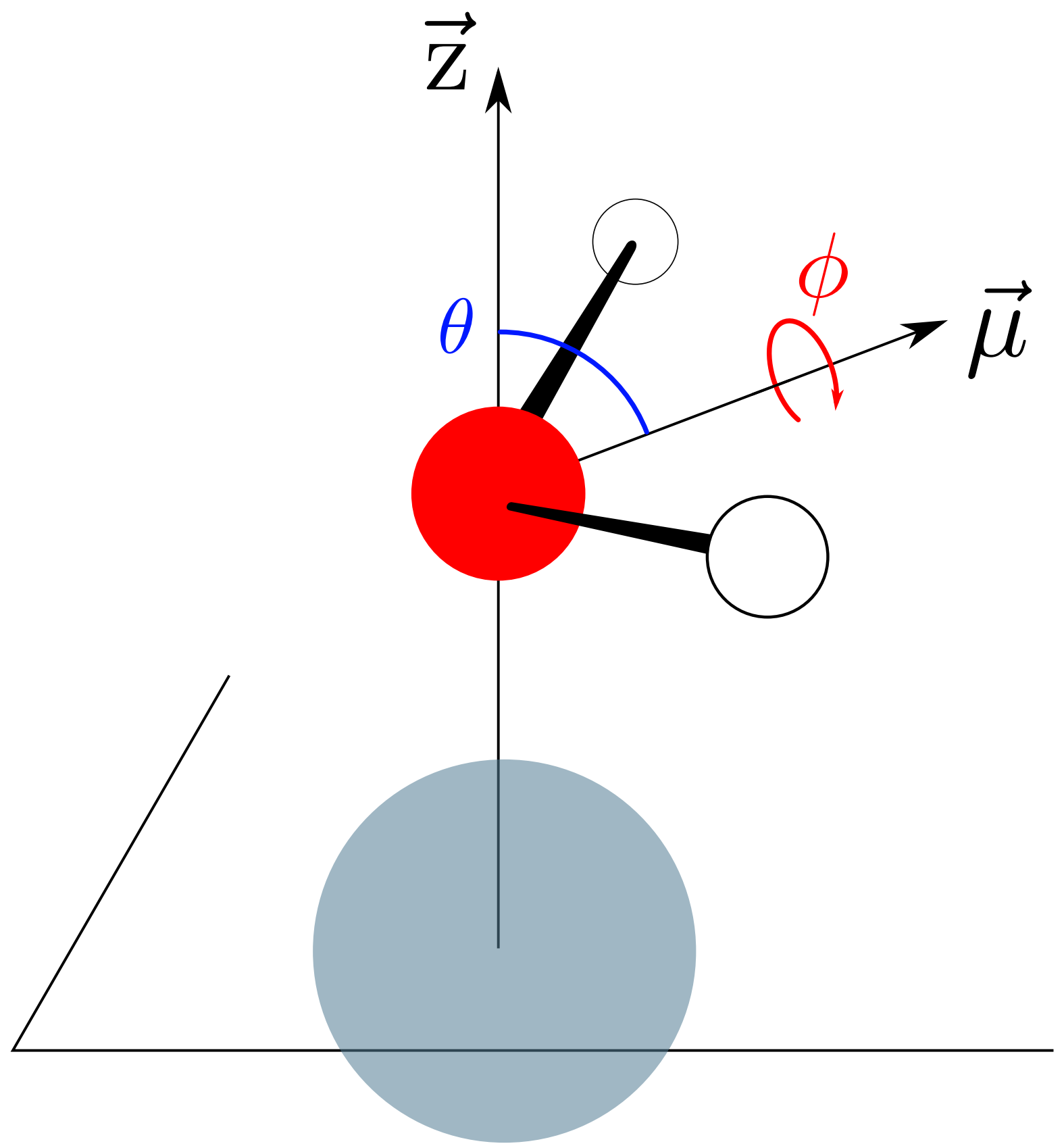

Figure S4: Representation of the cartwheel $(\theta)$ and propeller $(\phi)$ angles used to describe the orientation of water molecules toward the surface. $\mu$ is the dipol of the water molecule. $\theta=0^{\circ}$ when $\mu$ is aligned with the $\mathrm{z}$ axis, $\phi=0^{\circ}$ when he two hydrogen are at an equal distance to the surface. 


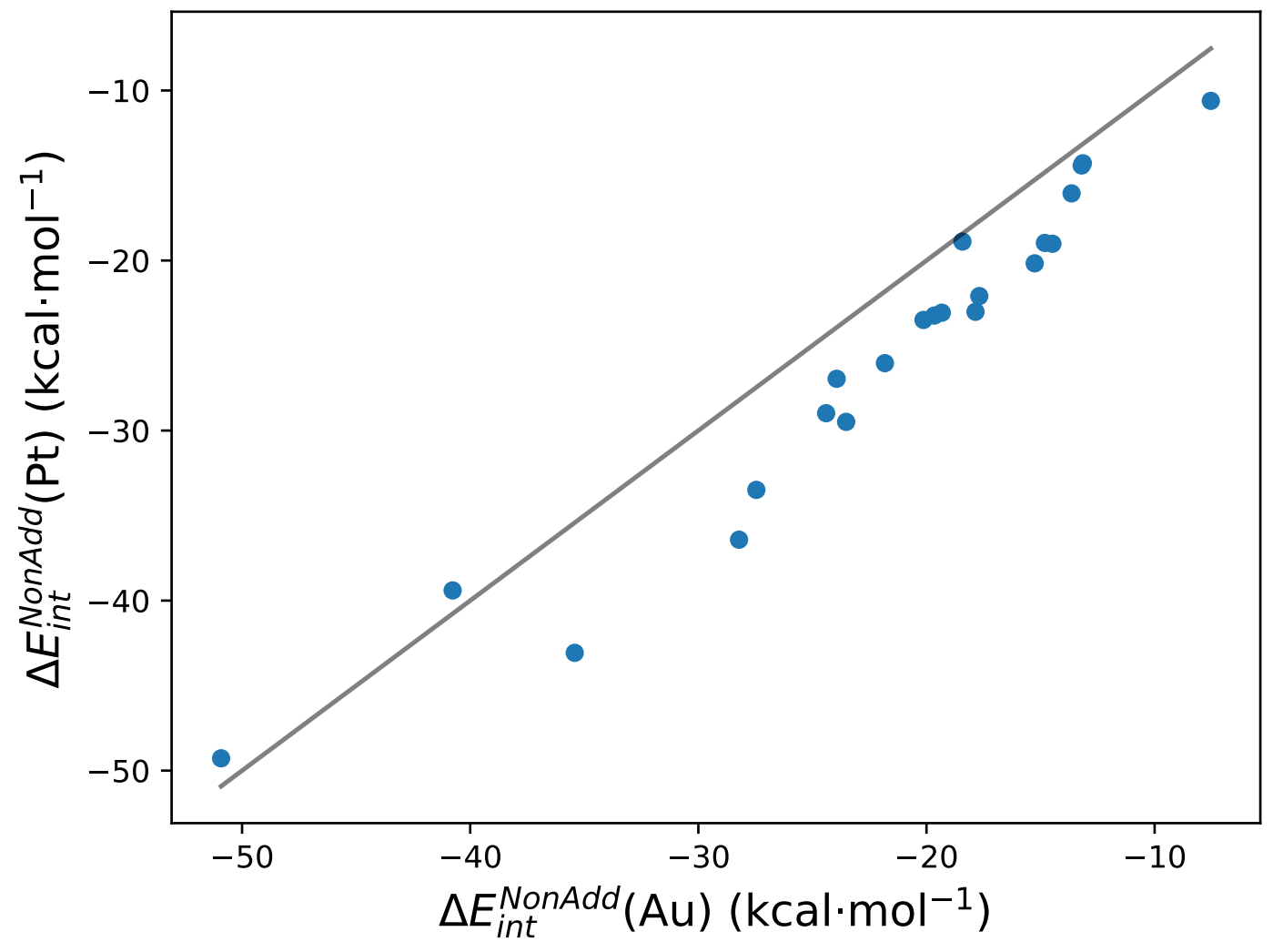

Figure S5: Correlation between $\Delta E_{\text {int }}^{\text {NonAdd }}$ for oligomers on $\mathrm{Pt}(111)$ vs $\mathrm{Au}(111)$. The black line corresponds to the bisector. 


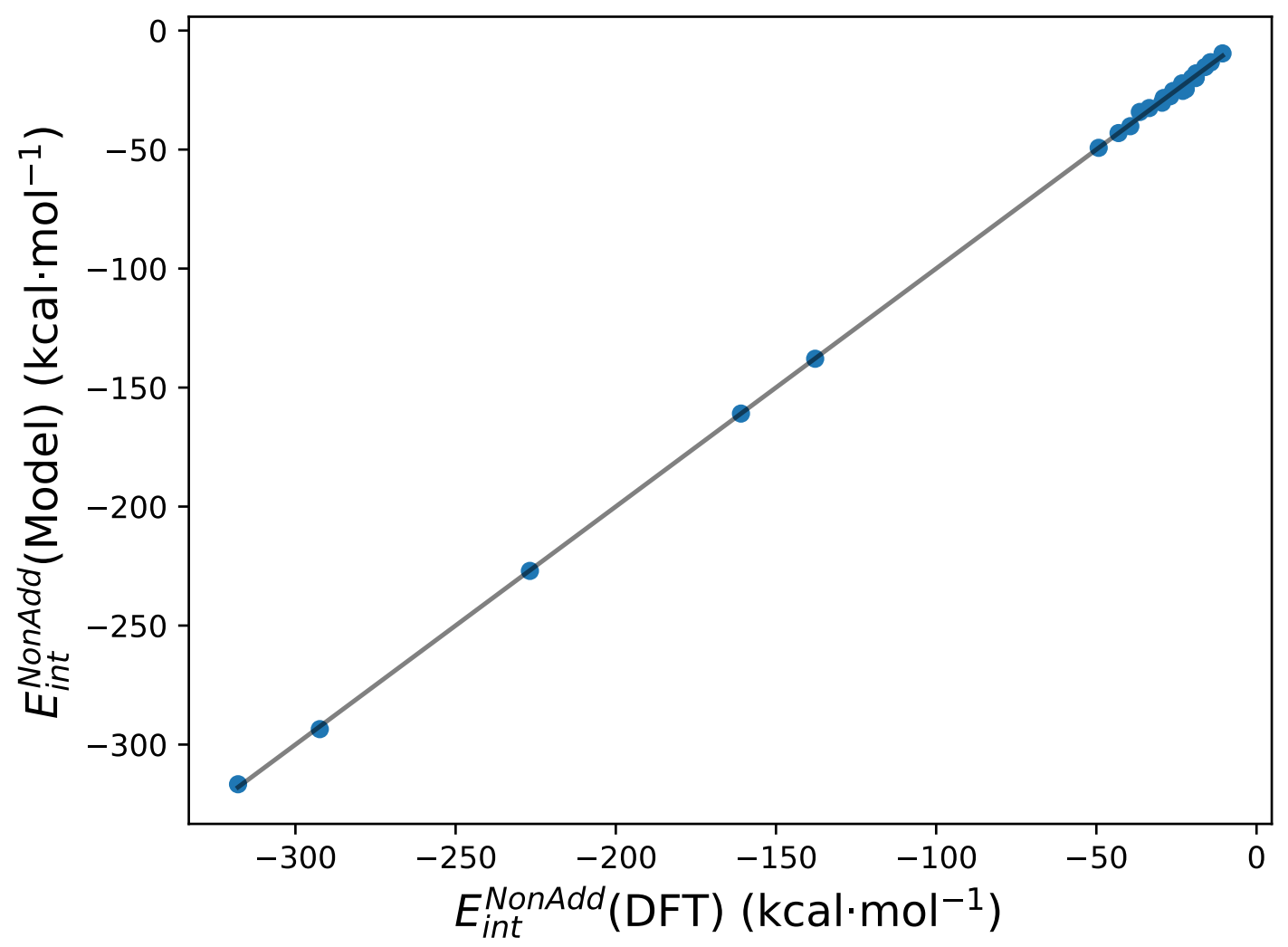

Figure S6: Parity plot for the linear regression model based on the H-bond acceptor type for the 27 investigated systems. Eleven variables are used (see Table S1), leading to a root mean squared error of $1.37 \mathrm{kcal} / \mathrm{mol}$. The black line represent the parity line. 


\section{References}

(S1) Heinz, H.; Vaia, R. A.; Farmer, B. L.; Naik, R. R. Accurate Simulation of Surfaces and Interfaces of Face-Centered Cubic Metals Using 12-6 and 9-6 Lennard-Jones Potentials. J. Phys. Chem. C 2008, 112, 17281-17290. 
Other files

imecs.tar.gz (14.15 KiB)

view on ChemRxiv - download file 